\title{
A review on metal additive manufacturing: modeling and application of numerical simulation for heat and mass transfer and microstructure evolution
}

\author{
Chuan-ming Liu, Hua-bing Gao, Li-yu Li, Jian-dong Wang, Chun-huan Guo, *Feng-chun Jiang \\ Key Laboratory of Superlight Materials \& Surface Technology, Ministry of Education, College of Materials Science and Chemical Engineering, \\ Harbin Engineering University, Harbin 150001, China
}

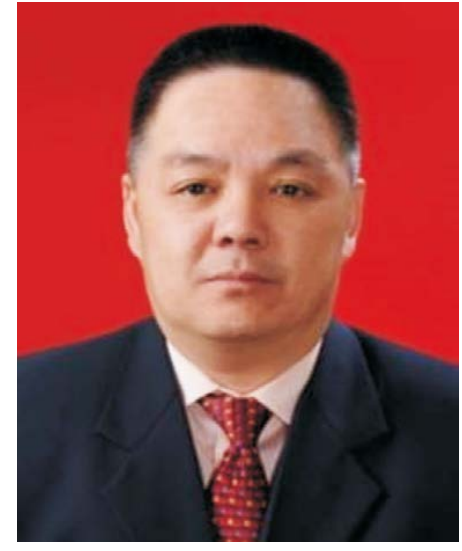

*Feng-chun Jiang

Professor Jiang is the Chief Scientist of National Key R\&D Plan Project (additive manufacturing and laser manufacturing), the Director of Advanced Materials and Manufacturing Technology Research Center of Harbin Engineering University. His research interests mainly focus on laser additive manufacturing technology and equipment, design and manufacture of metal layered composites and metal hollow sphere composites. He broke through the key technology of high-power transducer manufacturing, and developed the first high-power ( $9 \mathrm{~kW}$ ) ultrasonic rapid forming and additive manufacturing equipment in China. His group creatively integrated highpower ultrasonic machining technology into metal additive manufacturing. Professor Jiang possesses 16 authorized patents of China, published more than 160 academic papers, and won three provincial and ministerial-level scientific and technological progress awards.

E-mail: fengchunjiang@hrbeu.edu.cn
Abstract: Metal additive manufacturing technology has been widely used in prototyping, parts manufacturing and repairing. Metal additive manufacturing is a multi-scale and multi-physical coupling process with complex physical phenomena of heat and mass transfer and microstructure evolution. It is hard to directly observe the dynamic behavior and microstructure evolution of molten pool during additive manufacturing. Therefore, numerical simulation of additive manufacturing process is significant since it can efficiently and pertinently predict and analyze the physical phenomena in the process of metal additive manufacturing, and provide a reference for technological parameters selection. In this review, the research progress of numerical simulation of metal additive manufacturing is discussed. Various aspects of numerical simulation models are reviewed, including: (1) Introduction of basic control method and physical description of numerical simulation models; (2) Comparison of various heat and mass transfer models based on different physical assumptions (heat conduction model; heat flux coupling model; discrete powder particle heat flux coupling model); (3) Applications of various microstructure evolution models [phase field (PF), cellular automata (CA), and Monte Carlo $(\mathrm{MC})$ ]. Finally, the development trend of numerical simulation of metal additive manufacturing, including the thermal-flow-solid coupling model and deep learning for numerical model, is analyzed.

Key words: additive manufacturing; numerical simulation; heat and mass transfer; microstructure evolution

CLC numbers: TP391.9; Document code: A;

Article ID: 1672-6421(2021)04-317-18

\section{Introduction}

Metal additive manufacturing technology is a new manufacturing technology with low cost, rapid prototyping, and convenient design ${ }^{[1,2]}$. Due to the great design friendliness, additive manufacturing technology has great application and development prospects in aviation, aerospace, nuclear power, medical and other fields that require the complex design of small-batch customization.

Although additive manufacturing technology has the above advantages, some problems may occur during the manufacturing process ${ }^{[3-6]}$. The main problems need to be solved in the additive manufacturing process include:

(1) It is difficult to control the thermal stress that leads to the cracking and deformation during the deposition process;

(2) Pores and micro cracks are easily produced in the internal surface of the component;

(3) Some mechanical properties of the parts may be deteriorated after deposition process comparing with traditional manufacturing technology.

The main reasons caused the defects (cracks, pores and large deformation) in the 
components are the concentrated energy beam, high energy density, and the fast-moving speed of the heat source, which lead to the discontinuity and instability of the molten pool and the complex microstructure ${ }^{[7]}$. In some cases, the mechanical properties of the component even cannot meet the requirements. Considering the above problems (large deformation and defects) during additive manufacturing process, the relationship of manufacturing process-microstructure evolutionmechanical properties ${ }^{[8]}$ were proposed to help optimizing the manufacturing parameters, controlling the geometry shape, and improving the forming quality and mechanical properties.

To precisely control the forming qualities, it is necessary to directly observe the manufacturing process. However, the existing observation methods are difficult to visually describe the physical process of additive manufacturing. Besides, the dynamic evolution processes of microstructure are even hard to be recorded due to the high environment temperature and the extremely fast evolution speed. Therefore, the observation of microstructure and the assessment on forming qualities can only be conducted after the manufacturing process. This greatly limits the investigation on the cause of defect and principle of microstructure evolution during additive manufacturing process.

The computer assisted numerical simulation technology is one of the methods to solve the above problems. Due to the development of numerical simulation technology and the corresponding computer technology, it is possible to predict and study the relationship among manufacturing processmicrostructure evolution-mechanical properties during additive manufacturing process ${ }^{[9]}$. The additive manufacturing process is a multi-scale (macro manufacturing process and microstructure evolution process) and multi physical field (temperature field, stress field and flow field) coupling process including temperature field and stress field on the macro scale, flow field of molten pool dynamics on the meso scale and crystallization dynamics on the micro scale ${ }^{[10]}$. The time scale of micro and meso scale processes is usually microsecond level, but the processing time of the parts often takes several hours or even days. This non-uniform time scale is the major difficulty for the numerical model design, at the same time, this is also the key point that the multi-scale simulation must be solved.

Although the numerical simulation method has difficulties on solving non-uniform time scale problem, comparing with the high cost and long period 'trial and error' experiments, it is still a high cost-performance and high efficiency method to study the metal additive manufacturing process.

This paper introduces the basic principles and application scopes of different calculation models for additive manufacturing. These models are generally divided into two categories, heat and mass transfer model and microstructure evolution model. The heat and mass transfer models are divided into continuum heat conduction model, continuum heat flux coupling model and discrete powder particle heat flux coupling model and stress field model (coupled with heat conduction model) according to the assumptions on the research subject. Microstructure evolution models are classified as phase field model, cellar automatic model and Monte Carlo method. The research progress of the above models is discussed in detail.

\section{Basic control method and physical description of numerical simulation model}

\subsection{Continuum heat conduction model}

In the heat conduction model based on the continuum hypothesis, the finite element method (FEM) is unusually employed to conduct thermal analysis at the component level. In this method, to reduce the amount of calculation and the number of control equations, the heat and mass transfer process in the molten pool is not explicitly simulated ${ }^{[11]}$. There are many assumptions and simplifications in this model, so the calculation accuracy is low. The temperature field model obtained by FEM can be used to further analyze thermal stress and deformation.

The basic control equation of heat conduction model is ${ }^{[12]}$ :

$\rho c \frac{\partial \phi}{\partial t}-\frac{\partial}{\partial x}\left(k_{x} \frac{\partial T}{\partial x}\right)-\frac{\partial}{\partial y}\left(k_{y} \frac{\partial T}{\partial y}\right)-\frac{\partial}{\partial z}\left(k_{z} \frac{\partial T}{\partial z}\right)-\rho Q=0$

where $\phi$ represents the field variable $(x, y, z, t)$ of transient thermal field, $\rho$ represents the density of the material, $c$ represents the specific heat capacity of the material, $T$ represents the system temperature, $t$ represents the system time, $Q$ represents the total heat input, $k$ is the thermal conductivity.

The boundary conditions include ambient temperature, heat source density, convective heat transfer and thermal radiation ${ }^{[12]}$.

$$
\begin{gathered}
T(x, y, z, t)=\bar{T}(t)\left(\Gamma_{1}\right) \\
k_{x} \frac{\partial T}{\partial x} n_{x}+k_{y} \frac{\partial T}{\partial y} n_{y}+k_{z} \frac{\partial T}{\partial z} n_{z}=\bar{q} f(t)\left(\Gamma_{2}\right) \\
k_{x} \frac{\partial T}{\partial x} n_{x}+k_{y} \frac{\partial T}{\partial y} n_{y}+k_{z} \frac{\partial T}{\partial z} n_{z}=\bar{h}_{\mathrm{c}}\left(T_{\mathrm{e}}-T\right)\left(\Gamma_{3}\right) \\
k_{x} \frac{\partial T}{\partial x} n_{x}+k_{y} \frac{\partial T}{\partial y} n_{y}+k_{z} \frac{\partial T}{\partial z} n_{z}=\varepsilon \sigma\left(T_{\mathrm{e}}^{4}-T^{4}\right)\left(\Gamma_{4}\right)
\end{gathered}
$$

where $\bar{T}(t)$ is the given temperature on the boundary $\Gamma_{1}, \bar{q} f(t)$ is the given heat source on the boundary $\Gamma_{2}, \bar{h}_{\mathrm{c}}$ is the heat transfer coefficient between the object on the boundary $\Gamma_{3}$ and the surrounding environment $T_{\mathrm{e}}, \varepsilon$ is the field emissivity, $\sigma$ is the Stefan-Boltzmann constant, and $n$ is the directional cosine of normal outside boundary.

Instead of solving the whole function, the complex problems were simplified into several simple ones to obtain approximate solutions. It is usually used to study the problems that are difficult to be solved by conventional experimental methods or obtain accurate solutions. 


\subsection{Continuum heat flux coupling model}

In the heat flow coupling model based on continuum assumption, the FVM (finite volume method) and FEM (finite element method) are usually employed to simulate the thermal analysis process in which the powder bed, powder flow or fuse droplet are assumed as continuum. By assuming the powder particles as a continuum, the discretization degree in the calculation system can be greatly reduced, and the calculation efficiency can be improved. Through the heat flow coupling analysis, the heat convection and heat conduction processes inside the molten pool can be well simulated, the change of molten pool morphology and the molten flow inside the molten pool can be analyzed ${ }^{[13]}$.

The balance equations in heat flux coupling calculation include mass conservation equation, momentum conservation equation and energy conservation equation ${ }^{[14]}$.

Mass conservation equation:

$$
\frac{\partial \rho}{\partial t}+\frac{\partial(\rho u)}{\partial x}+\frac{\partial(\rho v)}{\partial y}+\frac{\partial(\rho w)}{\partial z}=0
$$

Momentum conservation equation:

$$
\begin{aligned}
& \frac{\partial(\rho u)}{\partial t}+\frac{\partial(\rho u u)}{\partial x}+\frac{\partial(\rho u v)}{\partial y}+\frac{\partial(\rho u w)}{\partial z} \\
= & \frac{\partial}{\partial x}\left(\mu \frac{\partial u}{\partial x}\right)+\frac{\partial}{\partial y}\left(\mu \frac{\partial u}{\partial y}\right)+\frac{\partial}{\partial z}\left(\mu \frac{\partial u}{\partial z}\right)-\frac{\partial p}{\partial x}+S_{u} \\
& \frac{\partial(\rho v)}{\partial t}+\frac{\partial(\rho v u)}{\partial x}+\frac{\partial(\rho v v)}{\partial y}+\frac{\partial(\rho v w)}{\partial z} \\
= & \frac{\partial}{\partial x}\left(\mu \frac{\partial v}{\partial x}\right)+\frac{\partial}{\partial y}\left(\mu \frac{\partial v}{\partial y}\right)+\frac{\partial}{\partial z}\left(\mu \frac{\partial v}{\partial z}\right)-\frac{\partial p}{\partial y}+S_{v} \\
& \frac{\partial(\rho w)}{\partial t}+\frac{\partial(\rho w u)}{\partial x}+\frac{\partial(\rho w v)}{\partial y}+\frac{\partial(\rho w w)}{\partial z} \\
= & \frac{\partial}{\partial x}\left(\mu \frac{\partial w}{\partial x}\right)+\frac{\partial}{\partial y}\left(\mu \frac{\partial w}{\partial y}\right)+\frac{\partial}{\partial z}\left(\mu \frac{\partial w}{\partial z}\right)-\frac{\partial p}{\partial z}+S_{w}
\end{aligned}
$$

Energy conservation equation:

$$
\begin{aligned}
& \frac{\partial(\rho T)}{\partial t}+\frac{\partial(\rho u T)}{\partial x}+\frac{\partial(\rho v T)}{\partial y}+\frac{\partial(\rho w T)}{\partial z} \\
= & \frac{\partial}{\partial x}\left(\frac{k}{c_{\mathrm{p}}} \frac{\partial T}{\partial x}\right)+\frac{\partial}{\partial y}\left(\frac{k}{c_{\mathrm{p}}} \frac{\partial T}{\partial y}\right)+\frac{\partial}{\partial z}\left(\frac{k}{c_{\mathrm{p}}} \frac{\partial T}{\partial z}\right)+S_{T}
\end{aligned}
$$

where, $\rho$ is the density of liquid phase, $t$ is time, $u, v, w$ are the components of velocity vector in $x, y$ and $z$ directions, respectively. $S_{u}, S_{v}, S_{w}$ are the momentum source term in $z, y$ and $z$ directions. $c_{\mathrm{p}}$ is the material specific heat capacity, $T$ is the system temperature, $k$ is the fluid heat transfer coefficient, $S_{T}$ is the viscous dissipation term, $\mu$ is the viscous stress tensor.

The heat conduction model only assumes the thermal behavior of molten pool as the heat conduction. Comparing with the thermal conduction model, the fluid-thermal coupling model considers the thermal behavior inside the molten pool as the simultaneous reaction of heat convection and heat conduction. The boundary conditions of coupled heat flow calculation are composed of temperature field boundary conditions and flow field boundary conditions.

Temperature field boundary conditions:

$$
\begin{aligned}
& T(x, y, z, t)=\bar{T}(t)\left(\Gamma_{1}\right) \\
& k_{x} \frac{\partial T}{\partial x} n_{x}+k_{y} \frac{\partial T}{\partial y} n_{y}+k_{z} \frac{\partial T}{\partial z} n_{z}=\bar{h}_{c}\left(T_{\mathrm{e}}-T\right)\left(\Gamma_{3}\right) \\
& k_{x} \frac{\partial T}{\partial x} n_{x}+k_{y} \frac{\partial T}{\partial y} n_{y}+k_{z} \frac{\partial T}{\partial z} n_{z}=\varepsilon \sigma\left(T_{\mathrm{e}}^{4}-T^{4}\right)\left(\Gamma_{4}\right)
\end{aligned}
$$

The flow boundary conditions include the surface tension and recoil pressure at the gas-liquid interface ${ }^{[15,16]}$. Where the surface tension can be expressed as:

$$
F_{\mathrm{v}}=\left[\gamma_{\mathrm{m}}^{0}-\frac{\partial \gamma}{\partial T}\left(T-T_{\mathrm{m}}\right)\right] \boldsymbol{n} \boldsymbol{k}
$$

where, $\boldsymbol{n}$ is the normal vector of the interface and $k$ is the curvature of the gas-liquid interface, $\gamma_{\mathrm{m}}^{0}$ is the surface tension coefficient of the material at the melting point, $\frac{\partial \gamma}{\partial T}$ is the change rate of material surface tension coefficient with temperature, $T_{\mathrm{m}}$ is the melting point of the material.

Recoil pressure:

$$
P_{\mathrm{v}}=0.54 P_{\mathrm{sta}} \exp \left(H_{\mathrm{v}} \frac{T-T_{\mathrm{v}}}{R T T_{\mathrm{v}}}\right)
$$

where, $P_{\text {sta }}$ is the static pressure, $H_{\mathrm{v}}$ is the latent heat of evaporation of the material, $T_{\mathrm{v}}$ is the evaporation temperature of the material and $R$ is the ideal gas constant. The recoil pressure is produced by the flow of the surface fluid driven by the evaporation of the fluid and mainly acts on the surface of the molten pool just below the heat beam. When the recoil pressure is greater than the surface tension, it is easy to cause splash and the surface broken of molten pool.

VOF (volume of fluid) is a common method to track the change of liquid surface in fluid thermal coupling model, which is often used to study the morphology change of molten pool. VOF method is an interface tracking method based on Euler grid ${ }^{[17,18]}$. In this method, the incompatible fluid components share a set of momentum equations, and the phase volume fraction is introduced to track the interface in the computational domain.

$$
p=\left\{\begin{array}{cl}
p_{\mathrm{A}} & c=0 \\
p_{\mathrm{A}}+\sigma \frac{\nabla a}{|\nabla a|}\left(a_{\mathrm{B}}-a_{\mathrm{A}}\right) & 0<c<1 \\
p_{\mathrm{B}} & c=1
\end{array}\right.
$$

where $p$ is the interfacial pressure, $a$ represents the phase volume fraction, $\mathrm{A} / \mathrm{B}$ represents phase $\mathrm{A} / \mathrm{B}$, and $c$ represents the ratio of the volume of a phase to the volume of the grid, $\sigma$ is the pressure at the interface. Figure 1 shows the schematic diagram of VOF method. 


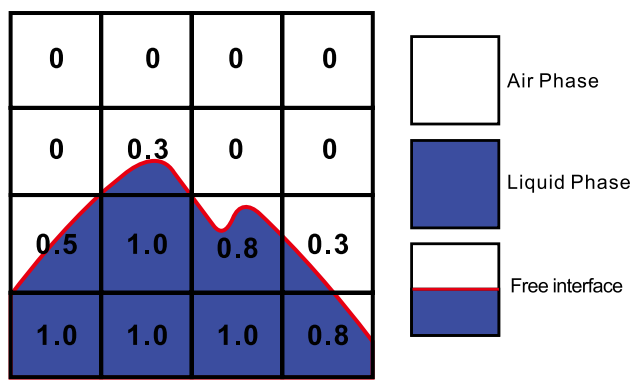

Fig. 1: Schematic diagram of VOF

The following assumptions are usually made in the momentum source term:

(1) Boussinesq approximation is used for the buoyancy term inside the molten pool ${ }^{[19]}$, dynamic buoyancy $\left(S_{\mathrm{F}}\right)$ can be expressed as:

$$
S_{\mathrm{F}}=g \beta\left(T-T_{\mathrm{m}}\right)
$$

where, $\beta$ is the coefficient of thermal expansion, $T_{\mathrm{m}}$ is the melting point of the material, and $g$ is the gravity coefficient.

(2) The mushy zone near the molten pool is simplified as a porous medium model, and the damping force of the liquid molten pool in the porous medium region is expressed by Darcy's formula ${ }^{[20]}$ :

$$
S_{\mathrm{D}}=-\frac{v}{K} \frac{1}{p_{1}} V
$$

where, $p_{1}$ is the density of material in liquid state, $K$ is the permeability of fluid in porous medium, $v$ is the inlet velocity, and $V$ is the velocity gradient of liquid phase.

Due to the influence of moving heat source, the surface temperature of molten pool does not distribute uniformly, which will produce surface tension gradient and promote the liquid metal to produce Marangoni convection in the molten pool. Marangoni effect is the main driving force for flow of molten pool ${ }^{[21]}$.

Considering that the high temperature in additive manufacturing process may lead to heat loss caused by evaporation of liquid metal, the heat flux boundary is as follows ${ }^{[22]}$ :

$$
q_{\text {evap }}=\frac{0.82 \Delta H_{\mathrm{v}}}{\sqrt{2 \pi M R T}} p_{0} \exp \left(\frac{\Delta H_{\mathrm{v}}\left(T-T_{\mathrm{v}}\right)}{R T T_{\mathrm{v}}}\right)
$$

where $q_{\text {evap }}$ is the heat loss due to evaporation, $M$ is the molar mass of the material, $H_{\mathrm{v}}$ is the latent heat of evaporation of the material, $T_{\mathrm{v}}$ is the evaporation temperature of the material, $R$ is the ideal gas constant and $p_{0}$ is the density of liquid metal.

\subsection{Discrete powder particle heat flux coupling model}

For heat flow coupling analysis based on powder particle scale, FVM, FEM, LBM (lattice Boltzmann method) and ALE (arbitrary Lagrange Euler) are usually utilized to discretize powder particles to simulate complex flow in molten pool and melting-solidification process of metal powder particles ${ }^{[23]}$. In this model, the coupled N-S equation, and the convection and diffusion equation are used to describe the mass and heat transfer in the micro-molten pool. High fidelity mesh discretization on powder scale can accurately consider the surface tension and Marangoni effect, so it is widely used to reveal the formation mechanism of manufacturing defects.

\subsection{Stress field model}

Stress field model usually couples with heat conduction model to help predict the stress-strain change during additive manufacturing process. The calculation of stress field model is generally based on thermoelastoplastic theory, including yield criterion, flow criterion and hardening criterion.

Von-Mises yield criterion is a commonly used yield criterion in metal structure analysis ${ }^{[24]}$ :

$\sigma_{i}=\sqrt{\frac{1}{2}\left(\sigma_{x}-\sigma_{y}\right)^{2}+\left(\sigma_{y}-\sigma_{z}\right)^{2}+\left(\sigma_{z}-\sigma_{x}\right)^{2}+6\left(\tau_{x y}^{2}+\tau_{y z}^{2}+\tau_{z y}^{2}\right)}$

where $\sigma_{x}, \sigma_{y}$ and $\sigma_{z}$ are the normal stress in different directions, $\tau_{x y}, \tau_{x z}$ and $\tau_{y z}$ are the shear stress on different planes, respectively.

The plastic strain increment derived from the plastic potential can be expressed as ${ }^{[25]}$ :

$$
\mathrm{d}\{\varepsilon\}_{p}=\mathrm{d} \lambda \frac{\partial \bar{\sigma}}{\partial\{\sigma\}}
$$

where $\lambda$ is plastic multiplier, $\varepsilon$ is the strain and $\delta$ is the stress.

Mises linear tracking hardening model is used as hardening criterion. The constitutive equation is based on the incremental theory:

$$
\{\mathrm{d} \sigma\}=[D]\{\mathrm{d} \varepsilon\}-\{C\} \mathrm{d} t
$$

where, $\mathrm{d} \sigma$ is the stress increment, $\mathrm{d} \varepsilon$ is the strain increment, $\mathrm{d} t$ is the time increment, $[D]$ is the elastoplastic matrix, and $\{C\}$ is the temperature dependent vector.

\subsection{Microstructure evolution model}

During the metal additive manufacturing process, the grain morphologies of three main primary solidification phases were observed. The various grain morphologies depend on the temperature gradient $(G)$ and solidification rate $(R)$ on the solidification front. $G / R$ is defined as a morphological parameter, and its value is related to solidification mode. For example, a relatively large $G / R$ value will generate columnar dendrites, and a small $G / R$ value will generate equiaxed dendrites; $G^{*} R$ is defined as cooling rate, which is related to grain size. The greater the cooling rate, the finer the grains ${ }^{[26]}$.

There are three kinds of numerical methods for solidification microstructure simulation in additive manufacturing process: deterministic method, randomness method and direct simulation method ${ }^{[27]}$.

In the deterministic method, the nucleation density and growth rate are determined as functions of supercooling, solute supersaturation and thermophysical parameters of materials. At the same time, the solid and liquid phase are divided according to the distribution of temperature field. The commonly used method for describing this phenomenon is molecular dynamics (MD) ${ }^{[28]}$. The randomness method 
reflects the nucleation and growth of crystals in a random way through the principle of probability theory. Monte Carlo (MC) method and cellular automata (CA) method are commonly used for randomness ${ }^{[29,30]}$. The direct simulation method describes the process of heat transfer, mass transfer and phase transition through unified mathematical and physical equations, and solves the solid-liquid interface in the process of numerical calculation. The commonly used direct simulation methods include level set method (LS) and phase field method (PF) ${ }^{[31,32]}$. The commonly used microstructure calculation models are phase field method, Monte Carlo method and cellular automata method.

Based on Ginzburg-Landau phase transition theory, the phase field method reflects the comprehensive effects of diffusion, ordering potential and thermodynamic drive in the physical process through mathematical and physical equations. In this method, the shape, curvature and evolution of the interface are described by the order parameters that are continuously changing in space. It does not need to track the interface, so the calculation accuracy is greatly improved, and the simulation results are closer to the physical mechanism of the actual problem. The phase field model could be coupled with solute concentration field and temperature field to simulate the solidification process ${ }^{[33]}$.

The phase field model considers the energy field as a whole system, and introduces $\varphi(r, t)$ order parameter to express the physical state of a system in space and time. $\varphi(r, t)=1$, it is solid phase, and $\varphi(r, t)=-1$ represents liquid phase. $\varphi(r, t)$ continuously change from -1 to 1 at the interface. According to the actual physical process, the corresponding free energy density function can be established. For a closed system, the expression of free energy can be expressed as ${ }^{[34]}$ :

$$
F(\varphi, T)=\int_{\mathrm{v}}\left[f(\varphi, c, e)+\frac{\varepsilon^{2}}{2}|\nabla \varphi|^{2}+\frac{\varepsilon_{c}^{2}}{2}|\nabla c|^{2}\right] \mathrm{d} V
$$

where $T$ is the current temperature of the phase, $T_{\mathrm{m}}$ is the phase transition temperature and $V$ is the phase volume. When $T=T_{\mathrm{m}}$, the free energy density of solid phase is equal to liquid phase. When $T<T_{\mathrm{m}}$, the former is smaller than the latter. $c$ is the alloy composition and $e$ is the internal energy, $\varepsilon$ is the coefficient of order parameter gradient term, $\varepsilon_{c}$ is the gradient coefficient of solute field. The free energy of the system tends to decrease during solidification.

According to Eq. (16), and introducing the interface dynamic coefficient, solute diffusion coefficient and thermal diffusion coefficient, the phase filed model could be described as ${ }^{[34]}$.

$$
\begin{aligned}
& \frac{\partial \varphi}{\partial t}=-M_{\varphi} \frac{\partial F}{\partial \varphi} \\
& \frac{\partial c}{\partial t}=-\nabla \cdot\left(M_{c} \nabla \frac{\partial c}{\partial \varphi}\right) \\
& \frac{\partial e}{\partial t}=-\nabla \cdot\left(M_{u} \nabla \frac{\partial e}{\partial \varphi}\right)
\end{aligned}
$$

where $M_{\varphi}, M_{c}$ and $M_{u}$ are the phase field parameters related to interface dynamic coefficient, solute diffusion coefficient and thermal diffusion coefficient, respectively.

The basic governing equation of phase field is as follows ${ }^{[34]}$ :

$$
\begin{aligned}
& \frac{\delta F}{\delta \varphi}=-\nabla\left[\frac{\delta F_{\mathrm{v}}}{\partial(\nabla \varphi)}\right]+\frac{\partial F_{\mathrm{v}}}{\partial \varphi} \\
& F_{\mathrm{v}}=f(\varphi, c, e)+\frac{\varepsilon_{c}^{2}}{2}|\nabla \varphi|^{2}+\frac{\varepsilon_{c}{ }^{2}}{2}|\nabla c|^{2} \\
& \frac{\partial \varphi}{\partial t}=-M_{\varphi}\left(-\varepsilon^{2} \nabla^{2} \varphi+\frac{\partial f(\varphi, c, e)}{\partial \varphi}\right)
\end{aligned}
$$

where $F_{\mathrm{v}}$ is the volume free energy.

The equations above are simultaneous to describe the comprehensive effect of diffusion, ordering potential and thermodynamic drive. Usually, the governing equations are used to directly simulate the formation of microstructure without distinguishing the solid-liquid phase and its interface. They are often used in the microstructure evolution process such as dendrite formation, coarsening and solid phase transformation during solidification, and have outstanding advantages in quantitative description of dendrite morphology.

CA method used for solidification simulation includes three parts: nucleation, growth and diffusion calculations. In the nucleation model, the random nucleation model proposed by Rappazt et al. ${ }^{[35]}$ is used to express the Gaussian distribution between the nucleation density $n$ and the undercooling degree $\Delta T$ :

$$
\frac{\mathrm{d} n}{\mathrm{~d}(\Delta T)}=\frac{n_{\max }}{\sqrt{2 \pi} \cdot \Delta T_{\sigma}} \exp \left[-\frac{1}{2}\left(\frac{\Delta T-\Delta T_{N}}{\Delta T_{\sigma}}\right)^{2}\right]
$$

where, $n$ is the nucleation density, $n_{\max }$ is the maximum nucleation density, $\Delta T$ is the supercooling degree, $\Delta T_{\sigma}$ is the standard deviation supercooling degree, $\Delta T_{N}$ is the maximum nucleation undercooling.

The relationship between nucleation density and undercooling degree can be expressed as follows:

$$
n(\Delta T)=\int_{0}^{\Delta T} \frac{\mathrm{d} n}{\mathrm{~d}(\Delta T)} \mathrm{d}(\Delta T)
$$

In one time-step, the increment of nucleation density can be expressed as:

$$
\delta n=n[\Delta T+\delta(\Delta T)]-n(\Delta T)=\int_{\Delta T}^{\Delta T+\delta(\Delta T)} \frac{\mathrm{d} n}{\mathrm{~d}(\Delta T)} \mathrm{d}(\Delta T)
$$

The nucleation probability can be expressed as:

$$
p=\delta n A_{\mathrm{c}}
$$

where, $A_{\mathrm{c}}$ is the area of a cell.

When the undercooling degree of a cell is greater than the critical undercooling degree and the nucleation probability is greater than a random number of $0-1$ at the same time, the cell will produce a nucleation transformation. These nucleation sites are randomly selected in the cell space that satisfies the basic conditions of nucleation. 
The grain growth model considers that each cell in the CA model of molten pool solidification has three possible states, liquid (solid fraction equal to 0 ), paste (solid fraction between 0 and 1 ) or solid (solid fraction equal to 1$)^{[35]}$. The solidification state of a cell depends on its solid fraction, which is determined by its nucleation position and growth state. The solute conservation equation on both sides of solid and liquid is as follows:

$$
\begin{gathered}
V_{n}^{*} C_{\mathrm{L}}^{*}(1-k)=\left[-D_{\mathrm{L}}\left(\frac{\partial C_{\mathrm{L}}}{\partial x}+\frac{\partial C_{\mathrm{L}}}{\partial y}\right)+D_{\mathrm{s}}\left(\frac{\partial C_{\mathrm{s}}}{\partial x}+\frac{\partial C_{\mathrm{s}}}{\partial y}\right)\right] \cdot \boldsymbol{n}^{*} \\
C_{\mathrm{s}}^{*}=k C_{\mathrm{L}}^{*}
\end{gathered}
$$

where, $V_{n}^{*}$ is the growth rate of the interface, $\boldsymbol{n}^{*}$ is the normal unit vector of the interface, $C_{\mathrm{L}}$ and $C_{\mathrm{s}}$ are the solute concentration in liquid and solid phase, $D_{\mathrm{L}}$ and $D_{\mathrm{s}}$ are the diffusion coefficients in liquid and solid phase, $k$ is the solute partition coefficients. $C_{\mathrm{s}}^{*}$ and $C_{\mathrm{L}}^{*}$ are the equilibrium concentration of solute near the interface (i.e., the concentration of solute in liquid and solid phase near both sides of the interface).

Dendrite tip growth is a process of transition from liquid phase or paste cell to solid phase. The solid fraction $\left(f_{\mathrm{s}}\right)$ in the cell is updated in each calculation time-step.

$$
\begin{aligned}
& f_{\mathrm{s}}=f_{\mathrm{s}}^{0}+\delta_{\mathrm{f}} \\
& \delta_{\mathrm{f}}=\frac{\delta t}{\Delta x}\left(V_{x}+V_{y}-V_{x} V_{y} \frac{\delta t}{\Delta x}\right)
\end{aligned}
$$

where, $f_{\mathrm{s}}^{0}$ is the solid fraction of the last time-step of the cell, $\delta_{\mathrm{f}}$ is the increment of cell solid fraction in the current timestep, and $V$ is the growth rate, $t$ is the time.

Solute fraction is the key variable to calculate the growth rate of dendrite interfaces in the solute diffusion model. The solute fraction of the diffusion equation in the paste cell is expressed by an equivalent solute concentration:

$$
\begin{aligned}
& \frac{\partial C_{\mathrm{E}}}{\partial t}=\nabla \cdot\left(D_{\mathrm{E}} \nabla \cdot C_{\mathrm{E}}\right) \\
& C_{\mathrm{E}}=C_{\mathrm{L}}\left(1-f_{\mathrm{S}}\right)+C_{\mathrm{S}} f_{\mathrm{S}} \\
& D_{\mathrm{E}}=D_{\mathrm{L}}\left(1-f_{\mathrm{S}}\right)+C_{\mathrm{S}} f_{\mathrm{S}} k
\end{aligned}
$$

where, $C_{\mathrm{E}}$ and $D_{\mathrm{E}}$ are the equivalent solute fraction and the equivalent solute diffusion coefficient, respectively, $C_{\mathrm{L}}$ is the solute concentration in liquid phase and $D_{\mathrm{L}}$ is the diffusion coefficients in liquid phase, $f_{\mathrm{S}}$ is the solid fraction of the current step of the cell, and $k$ is the solute partition coefficients.

Monte Carlo model is a stochastic algorithm based on the principle of minimum free energy of grain boundary. In this algorithm, the whole computing area is discretized into regular lattices, and each lattice is assigned a grain identifier. If the grain identifiers of adjacent lattice are consistent, it means that they belong to the same grain, otherwise they belong to different grains. The total grain boundary energy of the system is expressed as ${ }^{[36]}$ :

$$
E=\frac{1}{2} \sum_{i=1}^{N} \sum_{j=1}^{L} 1-\delta\left(q_{i}, q_{j}\right)
$$

where $N$ is the total number of grids, $L$ is the total number of neighbors, $q_{i}, q_{j}$ are the identifier of lattice $i$ and $j . \delta$ is a Kronecker function.

The Monte Carlo method randomly selects two neighbor lattices on the grain boundary and adjusts their grain identifier to be consistent. If the change of free energy is less than $0(\Delta E<0)$, the modification will be accepted. Otherwise, the modification should follow a certain probability. In this situation, the model will generate a random number within the range of $(0,1)$. The random number will compare with the probability value of the following formula as the basis of whether the grain identification is modified or not.

$$
p=\left\{\begin{array}{l}
M(T), \Delta E \leq 0 \\
M(T) e^{-\frac{\Delta E}{k_{\mathrm{B}} T_{\mathrm{s}}}}, \Delta E>0
\end{array}\right.
$$

where $k_{\mathrm{B}}$ is Boltzmann constant, $T_{\mathrm{s}}$ is the temperature of Monte Carlo numerical calculation, $T$ is the temperature in physical sense, and $M(T)$ is the grain boundary mobility related to temperature, which is called grain boundary mobility.

\section{Research progress and application of numerical simulation to metal additive manufacturing}

\subsection{Temperature field and stress field distribution based on continuum heat conduction model}

Continum heat conduction model is usually used to calculate the overall temperature field distribution in additive manufacturing process. In the calculation process, the dynamics of molten pool is ignored and the powder bed or powder flow is assumed as continuum. The governing equation of the model takes the convection velocity in the energy conservation equation as 0 . Because there are few control equations in solving continuum heat conduction model, this model is widely used in solving large additive manufacturing model. The model is often coupled directly or indirectly with the thermal stress model to solve the problems of thermal deformation and thermal stress in the process of additive manufacturing.

The temperature field calculation results of heat conduction model are often used to calculate the thermal deformation and thermal stress of materials. The stress field in the additive process is obtained by direct, indirect or two-way coupling methods.

The heat conduction model based on continuum assumption is considered as a nonlinear transient heat conduction problem ${ }^{[37,38]}$ :

(1) It is assumed that the metal powder particles are continuous, equivalent and isotropic;

(2) Explicit method was not used for simulating mass and heat transfer in molten pool; 
(3) The volume loss caused by metal vapor or splash during additive manufacturing is not considered.

Current research for numerical simulation model of temperature field based on continuum heat conduction model focuses not only on the commonly used Gaussian heat source and double ellipsoid heat source, but also on the development of special heat source models and the optimization of heat source accuracy used in the model design.

Zhang et al. ${ }^{[26]}$ established a method based on mass balance to predict the clad geometry fabricated by high-power direct diode laser (HPDDL). Figure 2 shows the temperature evolution using HPDDL heat source model.
In Fig. 3, by tracing the temperature nodes on the pool boundary in the model, the thermal results such as the temperature history, temperature gradient, and solidification rate are investigated. Based on the simulated thermal results, the microstructure and Mo distribution in the clad layer were studied.

The HPDDL laser beam is rectangular with a top-hat distribution. At the focal plane, the size of the beam is $12 \mathrm{~mm} \times 3 \mathrm{~mm}$. The power distribution of the laser beam is expressed as ${ }^{[26]}$ :

$$
I(x, y, z, t)=\left\{\begin{array}{c}
\eta \frac{P}{w l}\left(-\frac{6\left(y-y_{c}\right)^{2}}{d^{2}}\right), 0.26 w \leq|x| \\
\eta \frac{P}{w l}\left(-\frac{6\left(y-y_{c}\right)^{2}}{d^{2}}\right)\left(0.3+\frac{1-0.3}{0.24 w}(0.5 w-|x|)\right), 0.26 \leq|x| \\
0 \text { for }|x| \geq 0.5 w
\end{array}\right.
$$

where $\eta$ is the laser absorptivity of material, $w$ and $l$ are the molten pool geometry
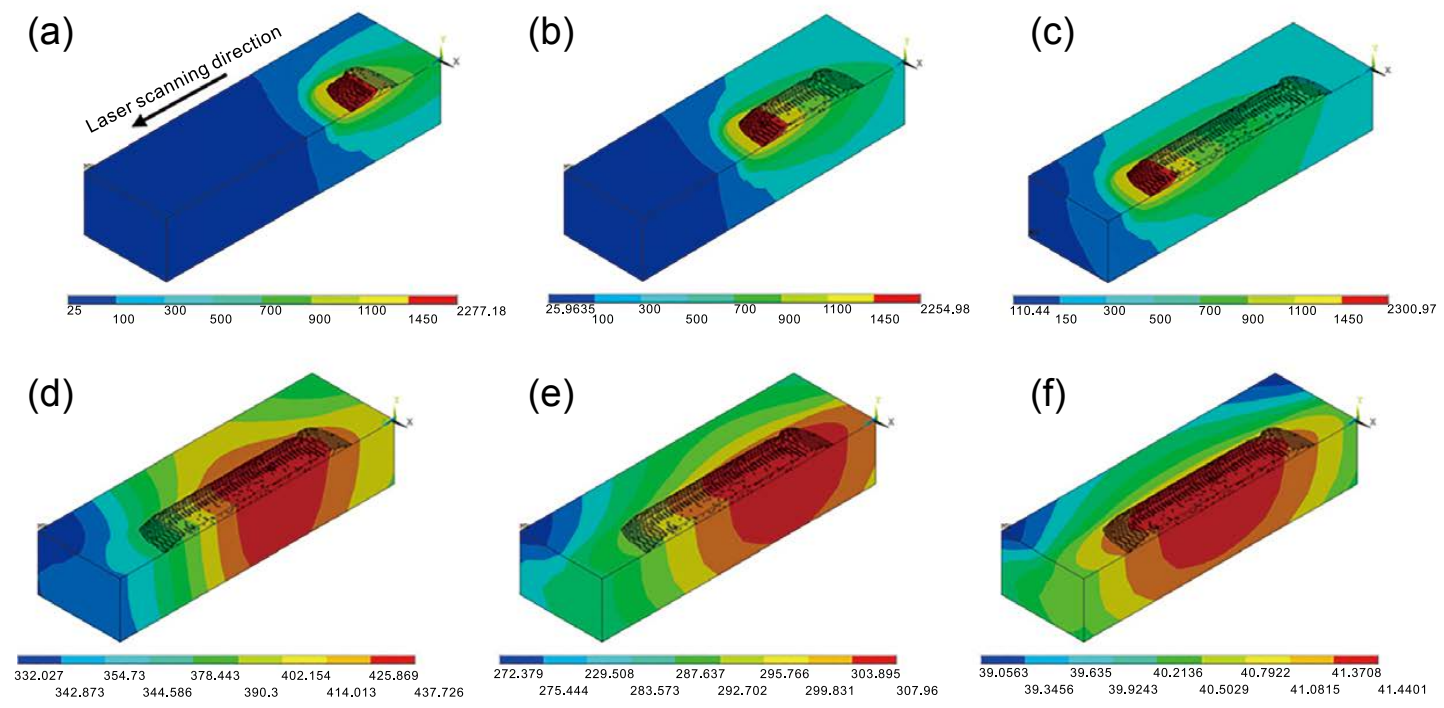

Fig. 2: Temperature evolution with power of $4,100 \mathrm{~W}$ and scanning speed of $3 \mathrm{~mm} \cdot \mathrm{s}^{-1}$ : (a) $4.9 \mathrm{~s}$; (b) $8.4 \mathrm{~s}$; (c) $13.4 \mathrm{~s}$; (d) $25 \mathrm{~s}$; (e) $50 \mathrm{~s}$; (f) $200 \mathrm{~s}^{[26]}$
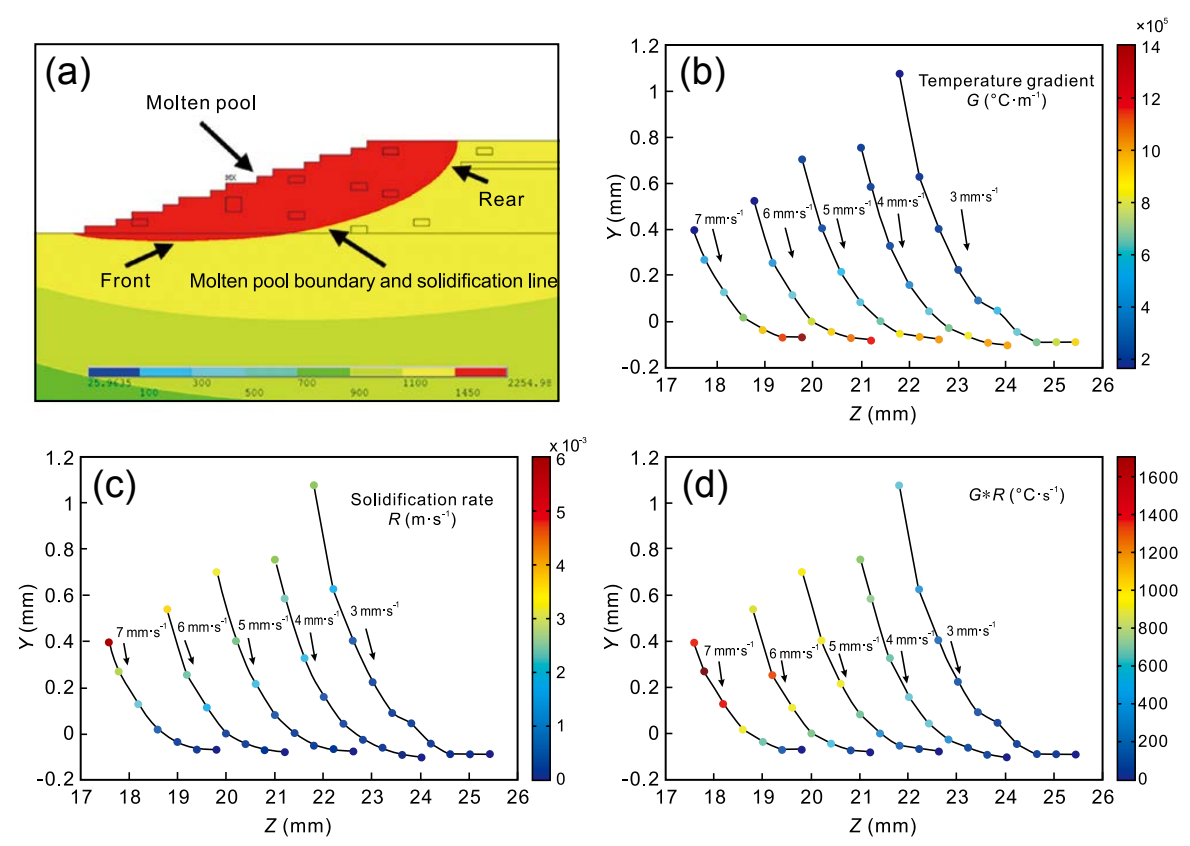

Fig. 3: Schematic of molten pool boundary (a) and distribution of $G(\mathrm{~b}), R(\mathrm{c})$, and $G^{\star} R(\mathrm{~d})^{[26]}$ 
parameter (width and length, respectively), $P$ is the laser power, and $d$ is the laser spot radius.

Some models evaluate the effect of convection on heat transfer in molten pool by modifying the heat transfer coefficient in molten pool.

Toyserkani et al. ${ }^{[39]}$ addressed a novel three-dimensional transient finite element model of the laser cladding by powder injection process. The proposed model can predict the clad geometry as a function of time and process parameters including beam velocity, laser power, powder jet geometry, laser pulse shaping, and material properties. The effect of fluid motion due to the thermocapillary phenomena can be taken into account using a modified thermal conductivity for calculating the molten pool boundaries. The effective thermal conductivity in the presence of thermocapillary flow is at least twice the stationary melt conductivity. This increase can be generally presented by ${ }^{[39]}$ :

$$
K^{*}(T)=a K\left(T_{\mathrm{m}}\right) \text { if } T>T_{\mathrm{m}}
$$

where $a$ is the correction factor, $K^{*}$ is the modified thermal conductivity, $T$ is the temperature and $T_{\mathrm{m}}$ is the melting point of the material.

In the heat conduction model, the effect of powder particles on temperature field can also be simulated by establishing the equivalent density of powder bed and its equivalent heat conduction coefficient. The calculation of equivalent density is relatively simple and can be determined via the powder density of powder bed. The calculation of equivalent heat transfer coefficient of powder bed is complex and there are many models.

Sih et al. ${ }^{[40]}$ established a simple model (Eq. 30) for calculating the emissivity and thermal conductivity of the surface of a powder bed through the porosity of powder bed and the emissivity of powder base metal. Estimation by the model was compared with experimental measurements for powder beds of alumina, silicon carbide, and iron. Agreement within the uncertainty of the measurements of $10 \%$ was obtained.

$$
\begin{gathered}
\frac{k_{\mathrm{e}}}{k_{\mathrm{g}}}=(1-\sqrt{1-\varepsilon})\left(1+\frac{\varepsilon k_{\mathrm{R}}}{k_{\mathrm{g}}}\right)+\sqrt{1-\varepsilon}\left(\frac{2}{1-\frac{k_{\mathrm{g}}}{k_{\mathrm{s}}}}\left(\frac{1}{1-\frac{k_{\mathrm{g}}}{k_{\mathrm{s}}}} \ln \frac{k_{\mathrm{s}}}{k_{\mathrm{g}}}-1\right)+\frac{k_{\mathrm{R}}}{k_{\mathrm{g}}}\right) \\
k_{\mathrm{R}} \approx 4 F \sigma_{\mathrm{sb}} T^{3} x_{\mathrm{R}}
\end{gathered}
$$

where $k_{\mathrm{e}}$ is the equivalent heat conduction coefficient of powder bed, $k_{\mathrm{g}}$ is the thermal conductivity of the gas in the powder gap, $k_{\mathrm{s}}$ is the thermal conductivity of the powder base metal, $\varepsilon$ is the porosity of the powder layer, $k_{\mathrm{R}}$ is the heat conduction coefficient produced by radiation, $F$ is $1 / 3$ apparent coefficient, $T$ is the temperature of powder, $x_{\mathrm{R}}$ is the effective length of thermal radiation between powder particles, usually the equivalent diameter of powder particles, $\sigma_{\mathrm{sb}}$ is the Stefan-Boltzmann constant.

It is an important application of continuum heat conduction model in practice to study the stress-strain change and residual stress distribution in additive manufacturing process by thermo-mechanical coupling model.

Li et al. ${ }^{[41]}$ developed a three-dimensional thermomechanical coupling model to simulate a multi-track, multilayer SLM (selective laser melting) process using the finite element method. The SLM scanning laser beam can be described as a moving volumetric heat source that is able to penetrate through the powder layers. The modeling results showed that the residual stress component of the built part in the direction of the layer height increased with the number of the printed layers. In Fig. 4, it can be found that at a given point, the residual stress component in the scanning direction is generally greater than the other two directions, and the maximum Von Mises stress occurs in the middle plane of the printed part.

The latent heat during melting and solidification is calculated by expressing the enthalpy and specific heat capacity of the material in solid state, solid-liquid mixture state and liquid state:

$$
h(T)=\left\{\begin{array}{c}
\int_{T_{\mathrm{r}}}^{T} \rho c_{\mathrm{s}}(T) \mathrm{d} T\left(T<T_{\mathrm{s}}\right) \\
\int_{T_{\mathrm{r}}}^{T_{\mathrm{s}}} \rho c_{\mathrm{s}}(T) \mathrm{d} T+\int_{T_{\mathrm{s}}}^{T}\left[\rho\left(\frac{\mathrm{d} L}{\mathrm{~d} T}\right)+\rho c_{\mathrm{m}}(T)\right] \mathrm{d} T\left(T_{\mathrm{s}}<T<T_{1}\right) \\
\int_{T_{\mathrm{r}}}^{T_{\mathrm{s}}} \rho c_{\mathrm{s}}(T) \mathrm{d} T+\rho L+\int_{T_{\mathrm{s}}}^{T_{1}} \rho c_{\mathrm{m}}(T) \mathrm{d} T+\int_{T_{1}}^{T} \rho c_{1}(T) \mathrm{d} T\left(T \geq T_{1}\right)
\end{array}\right.
$$

where $T$ is the temperature, $T_{\mathrm{r}}$ is the initial temperature, $T_{\mathrm{s}}$ is the solidus temperature and $T_{1}$ is the liquidus temperature. $c_{\mathrm{s}}$ is the specific heat capacity under solid state, $c_{\mathrm{m}}$ is the specific heat capacity under mixed state, and $c_{1}$ is the specific heat capacity under liquid state.

Some computational optimizations are also applied in the heat transfer model to improve the computational efficiency. A mesh coarsening strategy developed by Li et al. ${ }^{[42]}$ (Fig. 5) was used for predicting temperature, distortion, and residual stress in additive manufacturing, aiming to achieve feasible run time with reasonable accuracy on large builds. Two levels (a)

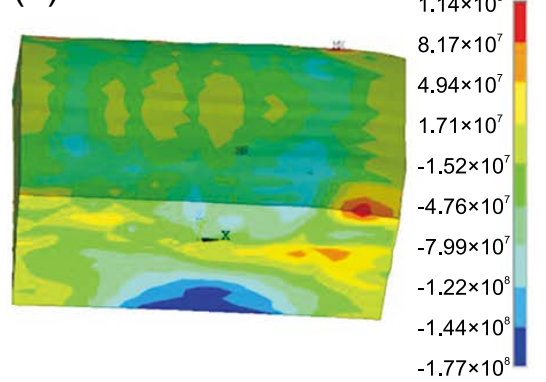

(b)

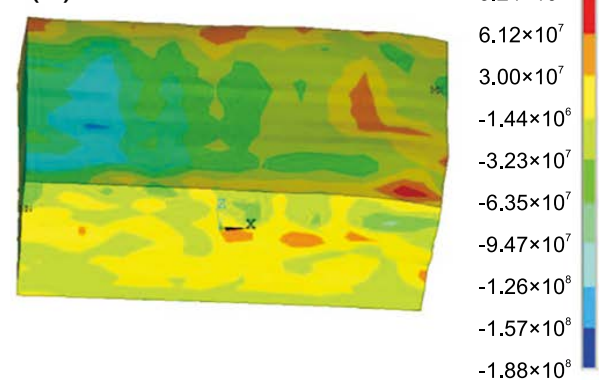

(c)



$9.47 \times 10^{7}$

$7.43 \times 10^{7}$

$5.40 \times 10^{7}$

$3.36 \times 10^{7}$

$1.33 \times 10^{7}$

$-7.09 \times 10^{6}$

$-2.74 \times 10^{7}$

$-4.78 \times 10^{7}$

$-6.81 \times 10^{7}$

$-8.85 \times 10^{7}$

Fig. 4: Distribution of normal residual stress for four-layer printed part in $X$ (a), $Y(b)$ and $Z$ direction (c) ${ }^{[41]}$ 


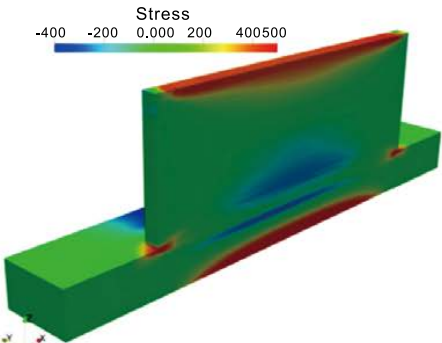

(a) Static mesh

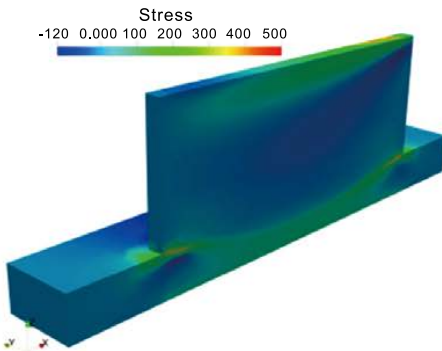

(d) Static mesh

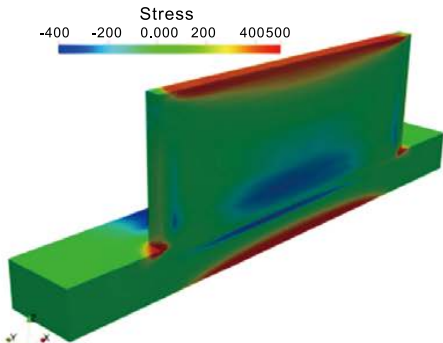

(b) Level 1 of coarsening

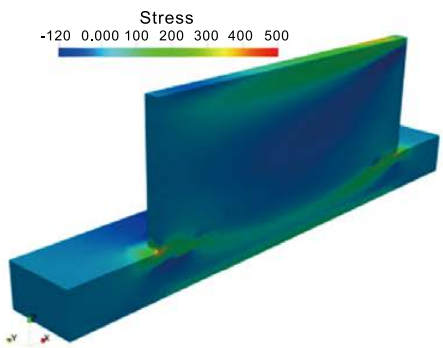

(e) Level 1 of coarsening

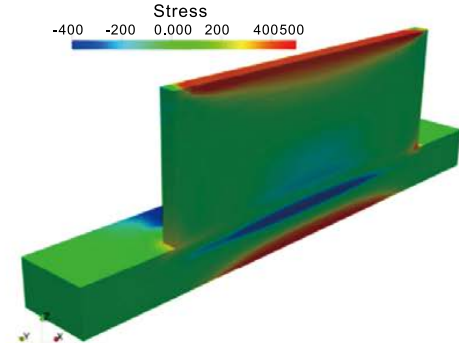

(c) Level 2 of coarsening,

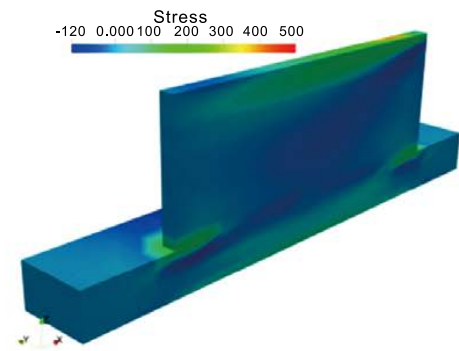

(f) Level 2 of coarsening

Fig. 5: Residual stress comparison of Inconel 625 (a-c) and Ti6Al4V (d-e) for different mesh coarsening levels: static mesh (a and d), Level 1 (b and e) and Level 2 (c and f) of mesh coarsening ${ }^{[42]}$

of mesh coarsening was found to be the most effective case. Comparing with the thermal simulation using static mesh strategy, this mesh strategy not only reduced the computational time by $75 \%$, but also got $2.5 \%$ less error for the peak distortion and negligible errors.

The results of the heat conduction model can be combined with the local micro stress-strain model to predict the possible microcracks in the process of additive manufacturing.

A stress-strain finite element model built by Wang et al. ${ }^{[43]}$ in fiber laser welding of 6013 aluminum alloy was developed based on the thermo-elastoplastic theory. Appropriate high temperature mechanical properties of aluminum alloy, the stress relaxation effect of the molten pool and the solidification shrinkage of filler metal were taken into consideration in this stress-strain field model. Figure 6 shows the distribution of longitudinal mechanical strains in brittle temperature region described using the thermo-elastoplastic model developed by Wang et al ${ }^{[43]}$.

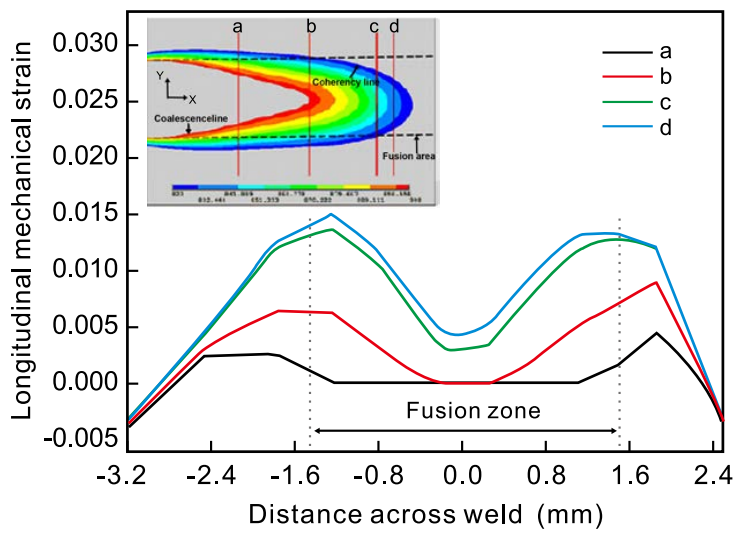

Fig. 6: Distribution of longitudinal mechanical strains in brittle temperature region ${ }^{[43]}$
Besides, a finite element model with respect to the local strain of columnar grains was developed considering the existence of liquid film (Fig. 7). Simulation results indicated that the magnitude of mechanical strain in liquid film was about $10^{-3}$ times as that in solid. The proposed model was used to simulate the initiation of solidification induced cracking under different welding speeds, and the critical pressure drop for crack initiation in laser welding of 6013 aluminum alloy was found.

\subsection{Melting and solidification behavior and morphology change of molten pool based on continuum heat flux coupling model}

Compared with the heat conduction model, the continuum heat flux coupling model can describe the shape and size of the molten pool and the heat convection in the molten pool more accurately. The powder, droplet and powder bed entering the molten pool are considered as equivalent continuum for heat flow coupling calculation. The basic assumptions of coupled heat flow model with continuum assumption include:

(1) The liquid phase in molten pool is considered as Newtonian and incompressible flow;

(2) The high energy beam is simplified as a heat source model;

(3) The mushy zone is assumed to be a homogeneous porous medium model.

The aforementioned model can accurately describe the Marangoni convection phenomenon in the molten pool and the molten pool deformation caused by the surface tension at the gas-liquid interface ${ }^{[44]}$.

Ogino et al. ${ }^{[45]}$ investigated the influence of deposition conditions during the WAAM (wire arc additive manufacturing) process on the shape of the deposition using a GMAM (gas metal arc welding) weld pool model. The results show that during the 
(a)

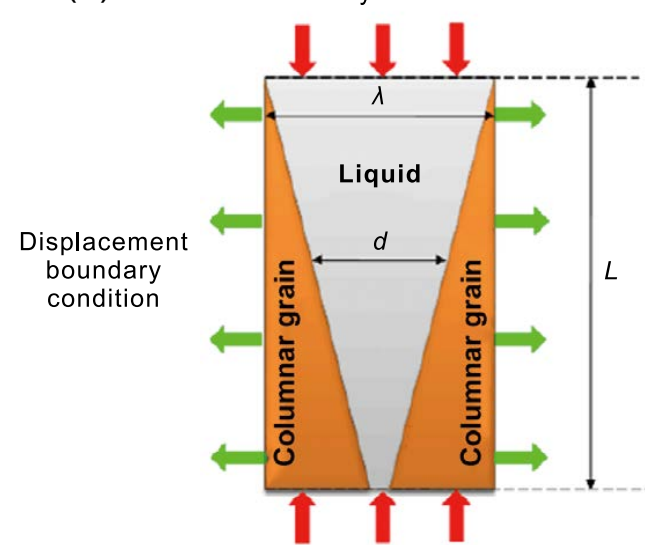

(b)

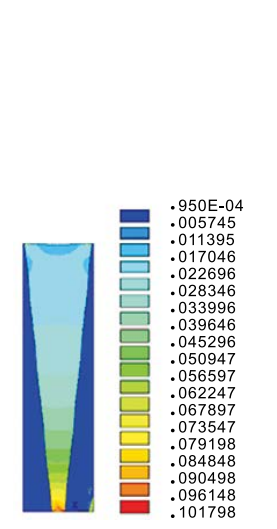

(c)

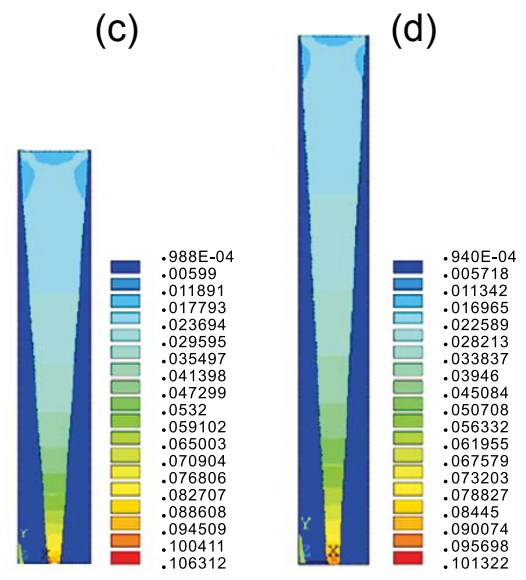

Fig. 7: Schematic of columnar grain FEM model (a) and effect of columnar grain length on local strain: (b) $250 \mu \mathrm{m}$; (c) $375 \mu \mathrm{m}$; (d) $500 \mu \mathrm{m}^{[43]}$

stacking process of straight wall parts, when there is cooling time between layers, the stacking layer will become higher and thinner, and the morphology of the parts with two adjacent layers stacked alternately is better than that with the same direction.

Zhang et al. ${ }^{[46]}$ established the numerical simulation model of arc additive manufacturing molten pool on convex surface. The simulation was based on FLUENT software. VOF (volume of fluid) method and enthalpy-porosity method were used respectively to track free surface of molten pool and melting solidification interface. Considering the influence of the droplet transition, adaptive double ellipsoid heat source, arc force and electromagnetic force models were established for simulating the dynamic deformation of molten pool surface. Figure 8 shows the molten pool surface evolution during WAAM process. The analysis results show that the arc additive manufacturing pool has a shape of low front and high back under the action of arc pressure, and the pool tends to flow on both sides when it is formed on the convex surface.

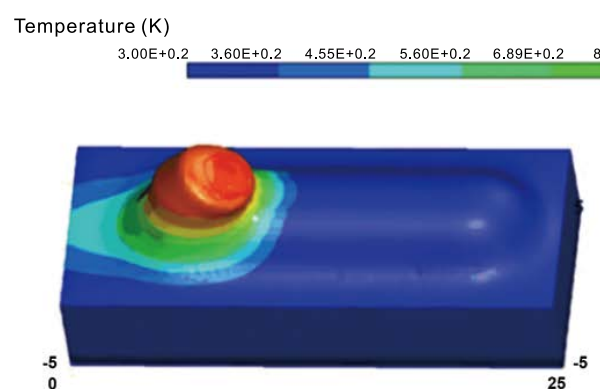

(a) $t=0.5 \mathrm{~s}$

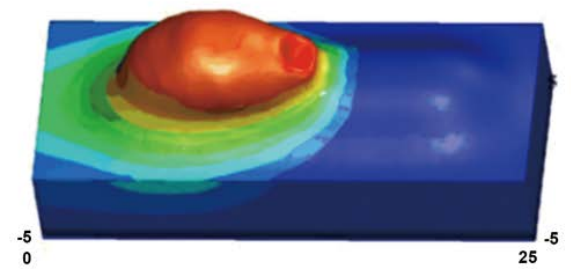

(c) $t=1.5 \mathrm{~s}$

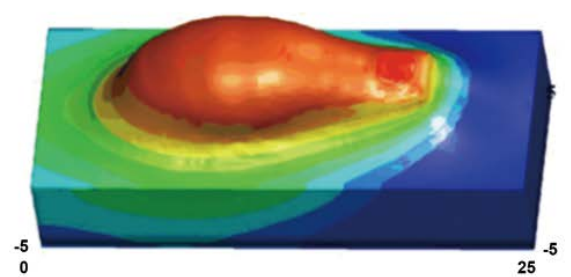

(e) $t=2.5 \mathrm{~s}$

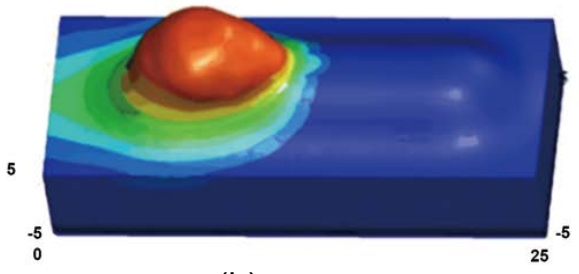

(b) $t=1 \mathrm{~s}$

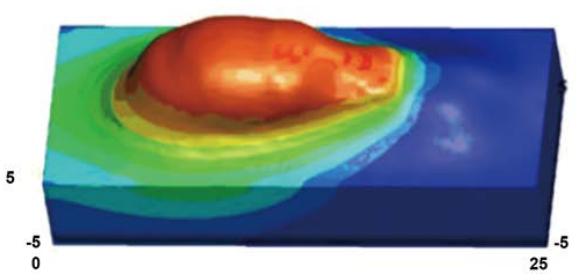

(d) $t=2 \mathrm{~s}$

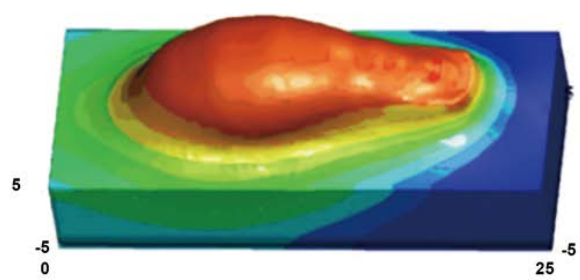

(f) $t=3 \mathrm{~s}$

Fig. 8: Molten pool surface evolution during WAAM process ${ }^{[46]}$ 
Yuan et al. ${ }^{[47]}$ completed a simulation of temperature evolution and thermal behavior of the molten pool during SLM of TiC/AlSi10Mg nanocomposites via FVM. The simulation results showed that Marangoni convection played a crucial role in intensifying the convective heat transfer and changing the molten pool geometry (Fig. 9). The temperature at laser-powder interaction zone, the molten pool dimensions and liquid lifetime increased with increasing the laser power or decreasing the scanning speed. The maximum temperature gradient within the molten pool increased significantly with increasing the applied laser power but increased slightly as a faster scanning speed was applied.
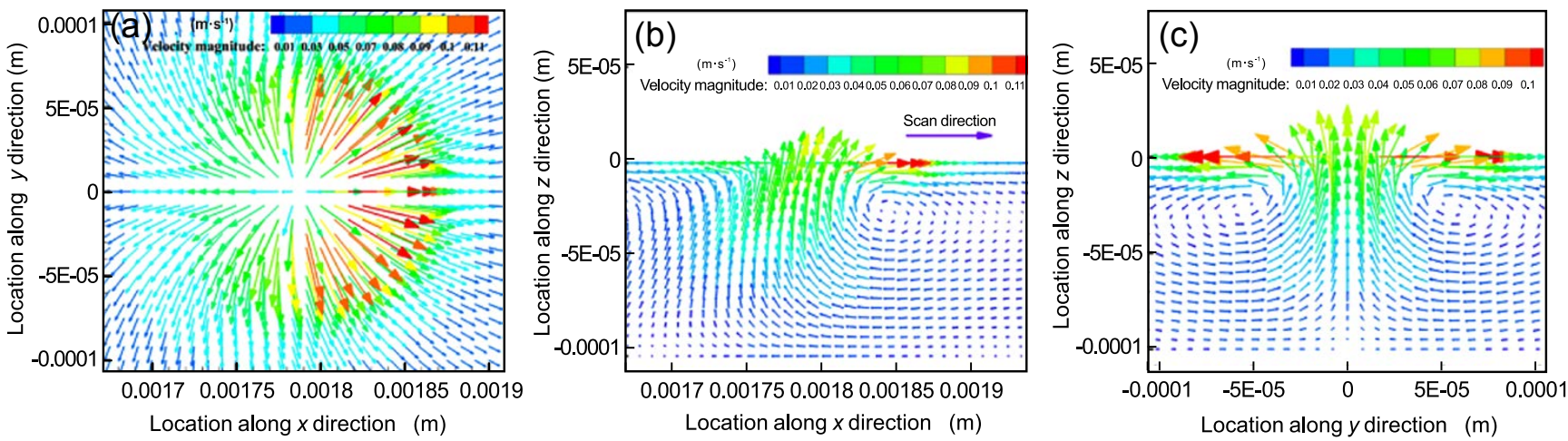

Fig. 9: Velocity vector plots in molten pool under action of Marangoni effect in different views: (a) top view; (b) longitudinal view at $Y=0$; (c) cross-sectional view at $X=1.5 \mathrm{~mm}$ (laser power $150 \mathrm{~W}$, scanning speed $400 \mathrm{~mm} \cdot \mathrm{s}^{-1}$, hatching space $50 \mu \mathrm{m}$, laser spot size $70 \mu \mathrm{m}$, scanning track 3) ${ }^{[47]}$

Similarly, the variation of powder particles in additive manufacturing process can be simulated by refining the heat conductivity and density of powder bed or powder flow in the continuum heat flow coupled model ${ }^{[48]}$ :

$$
\frac{k}{k_{\mathrm{f}}}=(1-\sqrt{1-\varphi})\left(1+\frac{\varphi k_{\mathrm{r}}}{k_{\mathrm{f}}}\right)+\sqrt{1-\varphi} \times\left\{\frac{2}{1-\frac{k_{\mathrm{f}}}{k_{\mathrm{s}}}}\left[\frac{1}{\left(1-\frac{k_{\mathrm{f}}}{k_{\mathrm{s}}}\right)^{2}}\left(1-\frac{k_{\mathrm{f}}}{k_{\mathrm{s}}}\right) \ln \left(\frac{k_{\mathrm{s}}}{k_{\mathrm{f}}}\right)-1\right]+\frac{k_{\mathrm{r}}}{k_{\mathrm{f}}}\right.
$$

where $\varphi$ is the porosity of the powder bed, $k_{\mathrm{f}}$ is the thermal conductivity of the fluid surrounding the powder and substrate, $k_{\mathrm{s}}$ is the thermal conductivity of the powder solid and $k_{\mathrm{r}}$ is the thermal conductivity portion due to the radiation among powder particles.
To study the effects of laser power and scanning speed on the thermal behavior and melting/solidification mechanism during SLM of TiC/ Inconel 718 powder system, Shi et al. ${ }^{[49]}$ employed the Eq. (32) to establish the model and study the size change of molten pool under different laser power and scanning speed, as shown in Fig. 10.
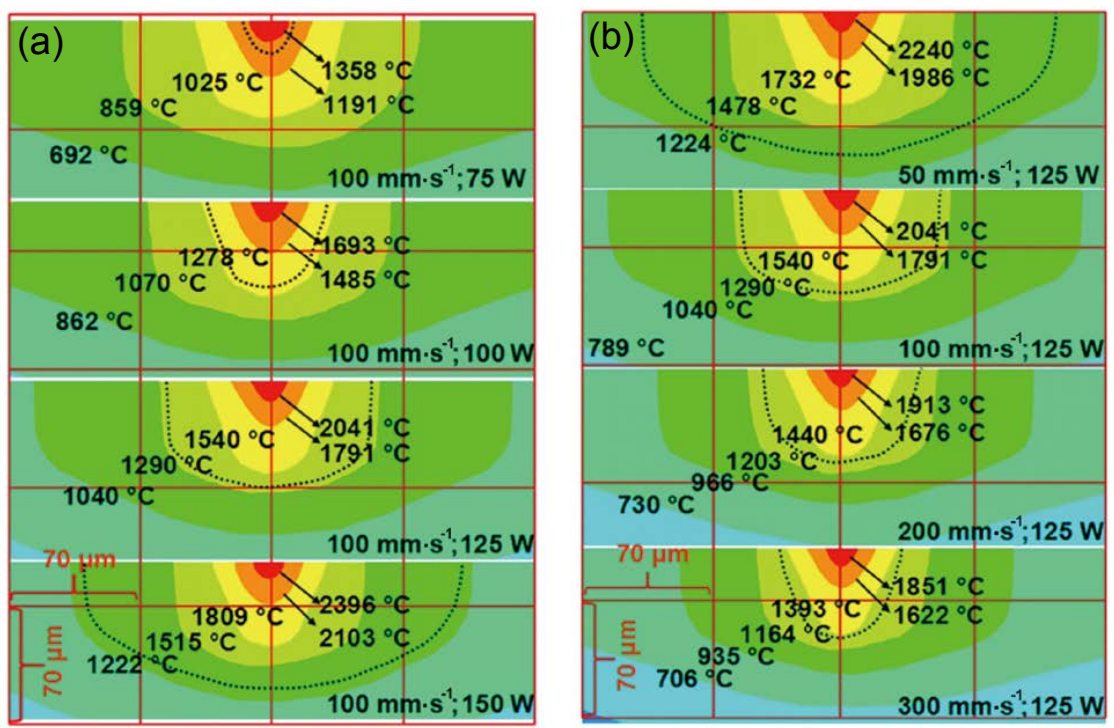

Fig. 10: Configuration of molten pool under different laser powers $\left(v=100 \mathrm{~mm} \cdot \mathrm{s}^{-1}, P=75 / 100 / 125 / 150 \mathrm{~W}\right)(\mathrm{a})$ and different scanning speeds $\left(P=125 \mathrm{~W}, v=50 / 100 / 200 / 300 \mathrm{~mm} \cdot \mathrm{s}^{-1}\right)(b)^{[49]}$ 
A predictive three-dimensional numerical model established by Gan et al. ${ }^{[50]}$ was developed to understand the multiphysical processes such as thermal behavior, Marangoni effect, composition transport, solidification behavior, and dendrite growth in multi-layer additive manufacturing of Ni-based alloy on cast iron substrate. Figure 11 shows the geometry of molten pool affected by temperature field and velocity field. According to the model considering the influence of temperature field and velocity field, this study indicates that the cooling rate declines progressively as the subsequent layers being deposited, which results in the coarser solidified grains in the upper part. Even though the powder and substrate can be efficiently mixed to be a homogeneous molten pool, a non-uniform concentration distribution is observed at the bottom of the deposited part, which agrees well with the experimental composition profile.

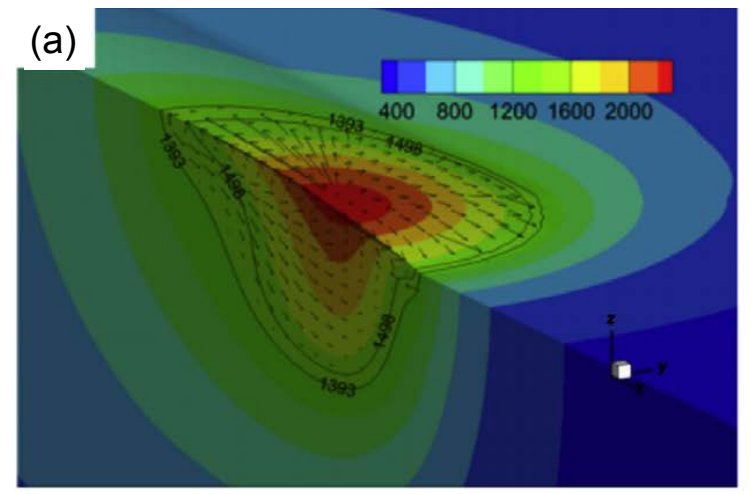

(b)

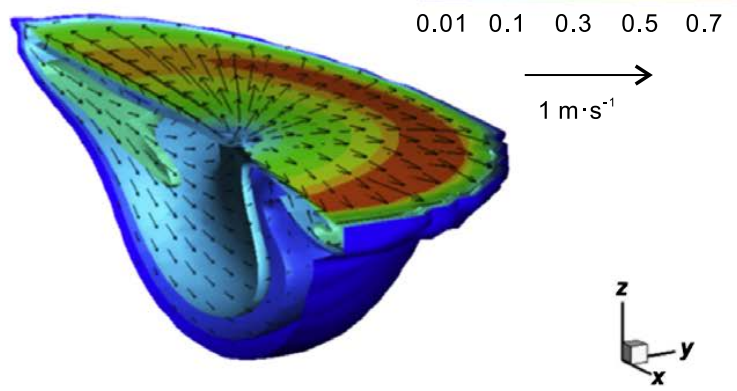

Fig. 11: Computed temperature (a) and velocity (b) distribution at $1,500 \mathrm{~ms}$ (at the mid-length of first layer) ${ }^{[50]}$

\subsection{Melting and flow behavior of metal particles and defect formation mechanism based on discrete powder particle heat flux coupling model}

The heat and mass transfer process in additive manufacturing process was simulated using discrete particle element method ${ }^{[52]}$. The model can accurately calculate the surface tension and Marangoni effect, so it is widely used to study the mechanism of defects in additive manufacturing process. DEM (discrete element method) is a commonly used method to describe random particles, which is developed from molecular dynamics theory ${ }^{[51]}$. Its basic principle is based on the interaction between particles and the Newton's law of motion, the force and displacement of particles at each time-step are determined by iterative calculation, and the macro motion law of particle flow is obtained by tracking the micro motion of a single particle. In one time-step, the solution process of DEM mainly includes four processes: contact break, contact force calculation, particle system motion update and boundary model motion update. Contact judgment is an extremely critical link in DEM simulation calculation. To improve calculation efficiency, it is generally not necessary to directly judge whether there is contact between any two particles, but to judge whether there is contact between particles in two steps: The first step is called neighbor search, which is to narrow the scope of particle search and determine the area around the detected particles and the particles under its jurisdiction; The second step is called intersection detection, which is to detect whether the particles in the area determined in the previous step are in real contact with the detected particles. In DEM, the contact deformation of particles is allowed to last for a certain period of time when they collide, and the collision deformation of particles is assumed to be the phenomenon that the shape of particles does not change when they collide, and a small amount of overlap occurs. The contact force between particles is calculated by overlap with other physical parameters, and the motion of particles is solved.

Chen et al. ${ }^{[53]}$ established a 3D finite difference method to predict the dynamic thermal behavior and flow mechanism of K418 powder irradiated by a Gaussian laser beam. A threedimensional randomly packed powder bed composed of spherical particles was established (Fig. 12) by DEM. The numerical results are in good agreement with the experiments, which proves that the numerical method is reasonable and effective. The results provide some in-depth insight into the complex physical behavior during SLM and guide the optimization of process parameters.

In the simulation, DEM was used to generate the powder particles, which contains the particle stack information (e.g., the size, shape, and coordinates of individual particles). This information was then input into the Flow-3D software to solve the thermal behavior and the fluid flow of the K418 materials during SLM by a developed 3D finite difference method (FDM) (Fig. 13).

LBM discretizes the particles velocity space, particles real space and the distribution function at the same time. A collision operator was used to simulate the evolution of the distribution function in a time-step, and then a lot of flow field information including density, pressure, internal energy and so on was calculated ${ }^{[54]}$. Absorption and reflection of laser by powder particles are shown in Fig. 14.

Zakirov et al. ${ }^{[55]}$ presented the results of $3 \mathrm{D}$ modeling of 

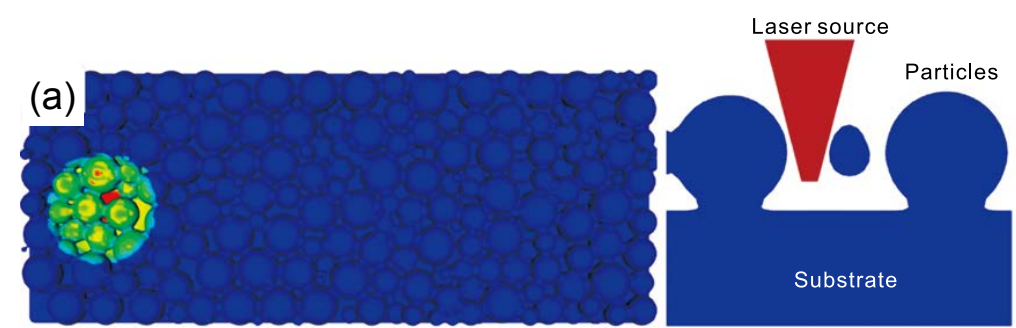

(b)

Surface tension
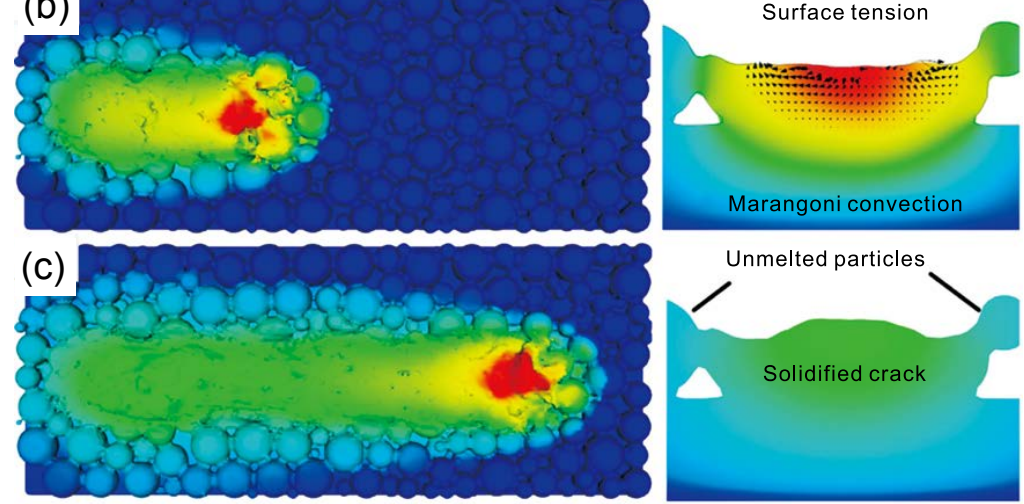

Fig. 12: Dynamic evolution of molten pool during SLM process of K418 at $P=320 \mathrm{~W}$ and $v=2,400 \mathrm{~mm} \cdot \mathrm{s}^{-1}$ : (a) absorption laser, (b) melting and flow, and (c) solidification ${ }^{[53]}$

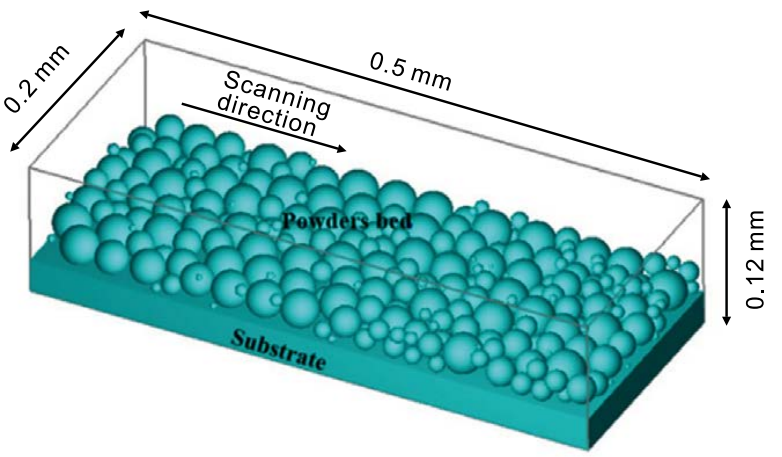

Fig. 13: Model of randomly packed K418 powder bed with Gaussian distributed particles ${ }^{[53]}$ the laser and electron beam powder bed fusion process at the mesoscale with a self-developed advanced multi-physical numerical tool. The hydrodynamics and thermal conductivity core of the tool is based on the lattice Boltzmann method. The numerical tool considers the random distributions of powder particles in a layer and the propagation of the laser (electron beam) with a full ray tracing (Monte Carlo) model. The ray tracing model includes multiple reflections, phase transitions, thermal conductivity, and detailed liquid dynamics of the molten metal.

ALE (Arbitrary Lagrangian Eulerian adaptive meshing) is a compromise between Eulerian description and Lagrangian description. In the Lagrangian description, the mesh nodes follow the deformation and motion points of the object

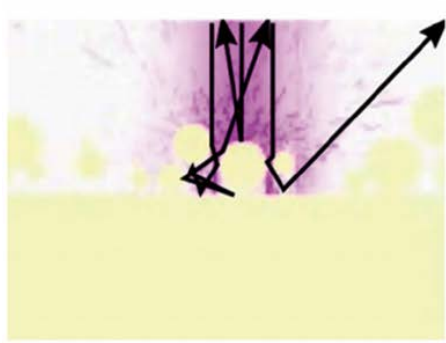

(a) No melting

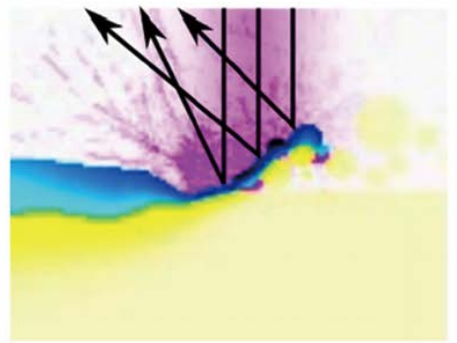

(b) Moderate melting

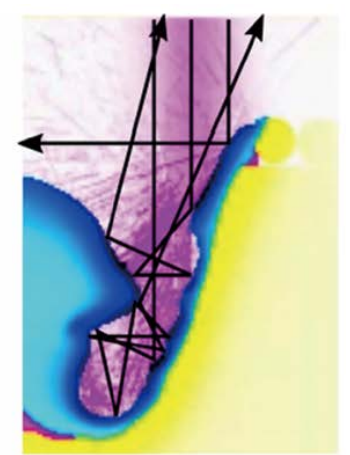

(c) Keyholing

Fig. 14: Different regimes of laser absorption during powder bed fusion process in 3D numerical simulations: cross-section along axis of laser movement ${ }^{[55]}$

completely, but the disadvantage is that the deformation cannot be too large. In Euler's description, grid nodes are fixed in space. Lagrange method focuses on the center of mass of fluid and tries to describe the motion process of each fluid particle from beginning to end, that is, the change of position with time.
Euler method focuses on spatial points and tries to express some physical quantities of fluid particles flowing through any fixed point in space at any time as a function of the position and time of the point ${ }^{[56,57]}$.

Based on the ALE-3D program (hybrid FVM and FEM) in the 
research of Khairallah et al. ${ }^{[58]}$, the powder scale high fidelity "thermal fluid solid" coupled numerical simulation was carried out. The random distribution powder bed was established by Particle Pack tool in ALE3D program. The Euler algorithm of ALE-3D partly solves the flow field and temperature field, and the Lagrange algorithm of ALE-3D solves the material motion and deformation. Figure 15 describes the melting, flow and solidification behavior of powder particles under laser effect and the interaction between particles.

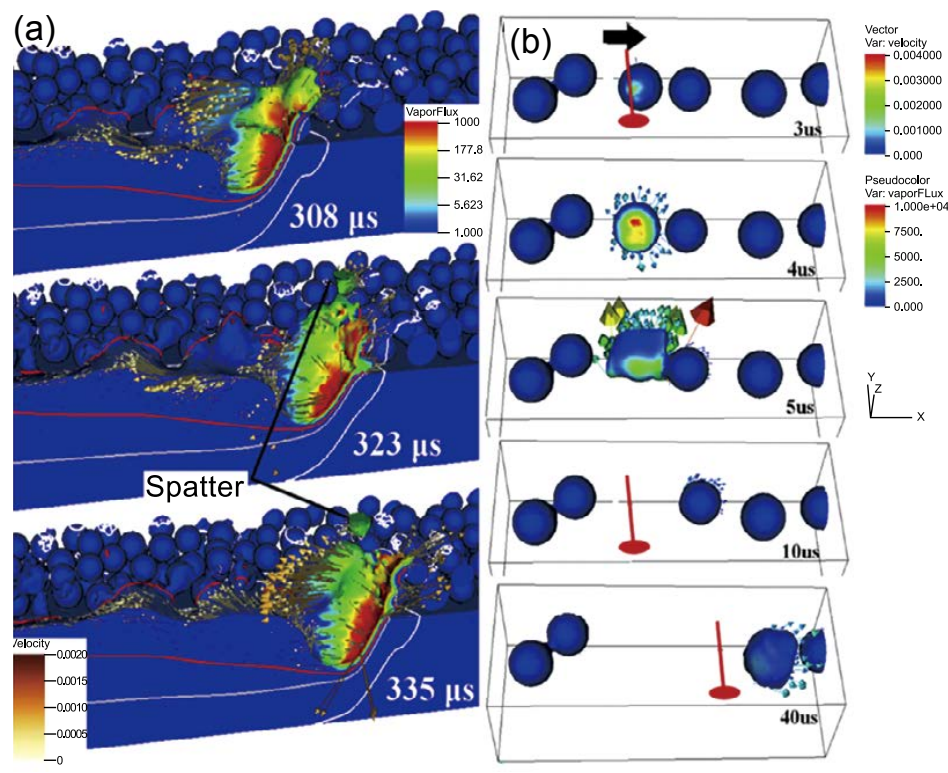

Fig. 15: Powder-scale finite element model simulations of laser powder bed fusion of metal powder ${ }^{[58]}$

\subsection{Modeling for solidification microstructure evolution under different calculation scales}

The forming quality and mechanical properties of parts in additive manufacturing are mainly determined by the internal microstructure of parts, so controlling the microstructure evolution process is the key in additive manufacturing technology. Additive manufacturing has the process characteristics of layer by layer accumulation. During the deposition process, the deposition layer will undergo several transient and unsteady thermal cycles, which makes the evolution of microstructure very complex.

PF method is usually used to solve two-dimensional crystal growth dynamics within three phases ${ }^{[59]}$. The solidification structure of Al-Cu alloy TIG weld was simulated by PF method, and the effect of welding speed on solidification microstructure was considered in the simulations. Results show that the cooling rate increases and the primary dendrite spacing decreases with increasing the welding speed. At the same time, the increase of welding speed increases the undercooling and makes the segregation more serious.

It is necessary to simulate topologically complex microstructure morphologies presented in laser powder deposition and other industrially relevant casting conditions. Fallah et al. ${ }^{[60]}$ combined finite element method with phase field to demonstrate the potential of coupling the phase-field method to complex heat transfer conditions. Figure 16 shows the dendrite growth at different positions of molten pool boundary simulated by means of phase field model. Figures 16(a) and (b) show
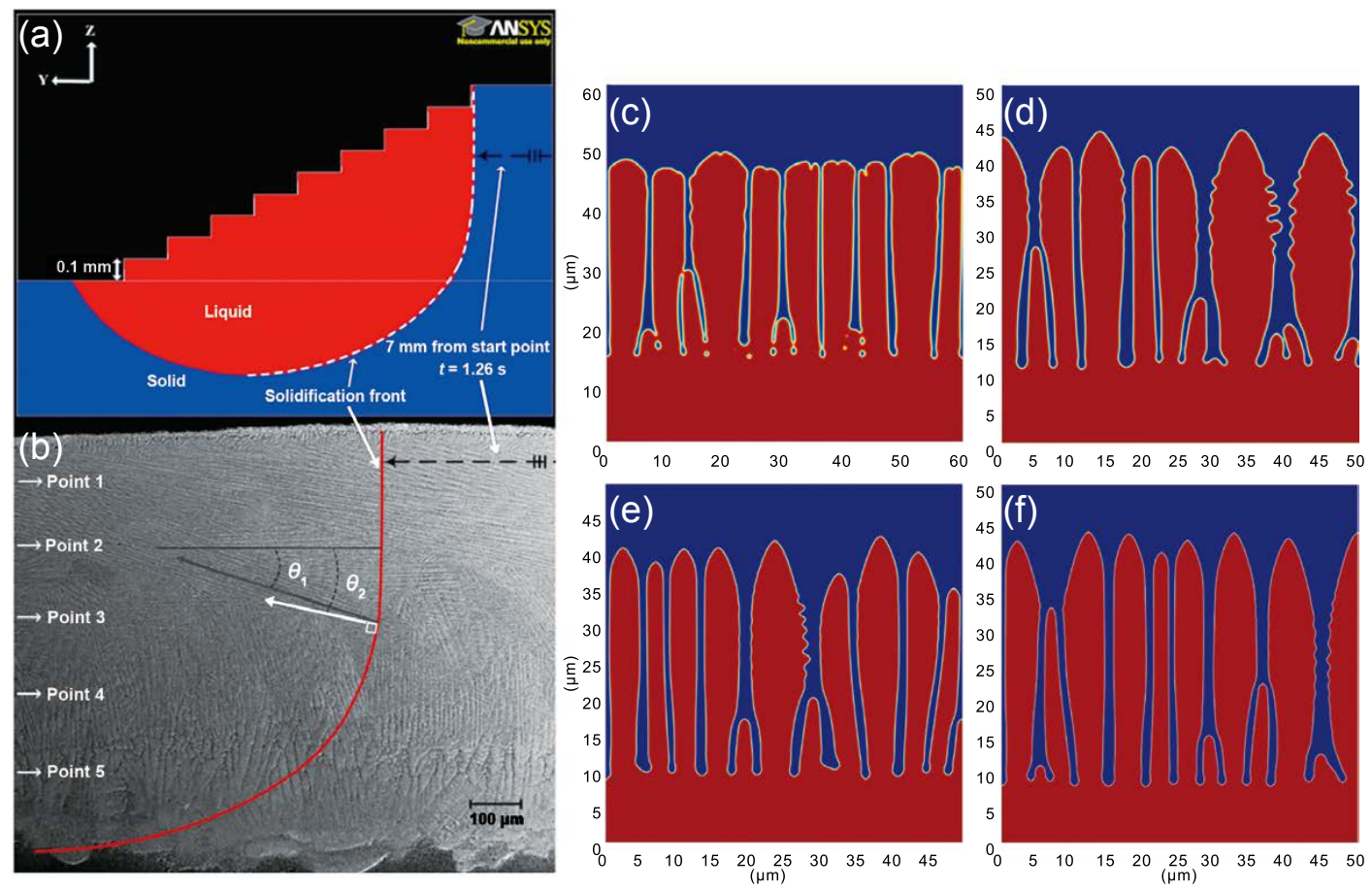

Fig. 16: Molten pool boundary simulated by FEM and effect of interface width on dendrite growth morphology ${ }^{[60]}$ 
the comparison between simulation result and experiment result. The melt pool boundary and the orientation of solidification front estimated by the isotherm of melting point is $1,735{ }^{\circ} \mathrm{C}$ at $1.26 \mathrm{~s}$. Comparing the predicted orientation of $\mathrm{S} / \mathrm{L}$ interface (the angle of normal to the red line and the horizon) $\theta_{2}$ with the experimentally revealed dendrite growth direction $\theta_{1}$ along the height of longitudinal section of the deposited material, the simulation result matches the experiment result well. The effect of interface width on dendrite growth morphology during directional solidification of Ti-32wt.\% Nb alloy under steady-state conditions [Figs. 16(c)(f)] is calculated by extracting the temperature gradient conditions obtained in the finite element model.

Liu et al. ${ }^{[61]}$ developed a multiscale model to investigate the evolution mechanisms of site-specific grain structures during additive manufacturing of alloys, taking the Ti-6Al-4V by selective electron beam melting (SEBM) as an example. It was found that the development of the predominant grain structures in the thick and thin walls, i.e., the large vertical columnar $<001>\beta / / \mathrm{Nz}$ grains and slanted columnar grains with various grain orientations, respectively, could be attributed to the competition and collaboration between the thermal gradient and the crystallographically preferred grain growth from the different growth stages in the simulations.

$\mathrm{CA}$ and $\mathrm{MC}$ methods are used to simulate grain growth by sum probability method, and the solid-liquid interface needs to be tracked for establishing the model. The CA method does not simulate the details of dendrite morphology. Instead, CA method simulates the dendrite contour by using the regular discrete grid to find the nucleation unit with the nucleation model. Generally, the mesh size of CA method is smaller than the secondary dendrite spacing of the problem studied, which leads to a huge amount of calculation and time-consuming in large-scale analysis ${ }^{[62]}$.

Kang et al. ${ }^{[63]}$ calculated microstructure evolution using a coupled FE-CA model, which contained FE model that simulated temperature field. Their results showed that the final microstructure of the welded joint included fine equiaxed grains in the equiaxed grains zone (EQZ), columnar in the fusion zone (FZ) and coarse equiaxed grains in the weld seam center zone. Moreover, it is indicated that the size of grains is associated with the temperature gradient of the welding process. Besides, the size of EQZ becomes narrow and uniform because that the increase of undercooling leads to the rise in the heat input. From Fig. 17, it could be found that the experiment fitted well the simulation result by the FE-CA model.

Mohebbi et al. ${ }^{[64]}$ developed a new approach to integrate a particle-based (seed crystals) nucleation mechanism into the CA simulation of powder-bed laser beam melting. The model aims to reflect two features observed in the microstructures of additive manufactured Al alloys: the fusion boundary nucleation, which violates the principle of common bulk nucleation, favored under high solidification rates and low thermal gradients, and the epitaxial growth at the melt-pool bottom as observed in the microstructure of AlSi10Mg alloy.

MC method refers to all statistical simulation algorithms using random numbers ${ }^{[65]}$. When MC method is used to

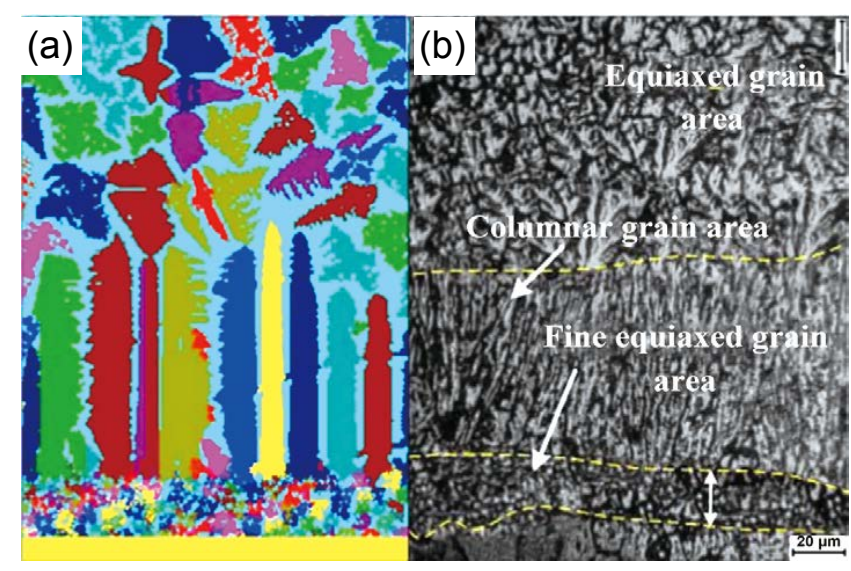

Fig. 17: Experimental (a) and simulated (b) results of 2219 LW seam microstructure ${ }^{[63]}$

simulate a physical system, the first thing is to describe the system with partial density function (PDF) or partition function (Z). Once the PDF or $\mathrm{Z}$ of the system is determined, the next step is to randomly extract "samples" from PDF, and then test the "samples" according to certain physical principles to get the results. After experiment, the output results are used to estimate the properties of the physical system or the behavior of the whole system.

Li et al. ${ }^{[66]}$ investigated the effects of laser transverse speeds on the microstructural morphology in directed energy deposition process. A Kinetic Monte Carlo (KMC) Potts model was used to exactly predict the grain evolvement under different laser scanning speeds, as shown in Fig. 18. Three increasing transverse speeds, 450, 600, and 1,000 $\mathrm{mm} \cdot \mathrm{min}^{-1}$, were investigated. The predicted microstructural morphology in depositions under three different laser transverse speeds was quantified and analyzed.

\section{Summary}

This paper summarizes the main heat and mass transfer models and their applications in the numerical simulation of metal additive manufacturing process.

The thermal conduction model is based on the heat conduction equation. This model ignores the metal melt flow in the molten pool. Therefore, it is simple for its small account of calculation. For the large-scale problems of component level, heat conduction model is the prior choice. This kind of model is described by Lagrange method, which is easily coupled with the thermal stress analysis model. Usually, thermal conduction model can quickly predict the distribution of residual stress and residual strain in the manufacturing process.

The heat flux coupling model is based on the thermal convection and thermal radiation equation. This model treats powder flow and powder bed as an equivalent continuum, which greatly reduces the discrete scale of the problem. Flow field boundary conditions and temperature field boundary conditions are combined to simulate the size of molten pool and temperature field considering the thermal convection. This 

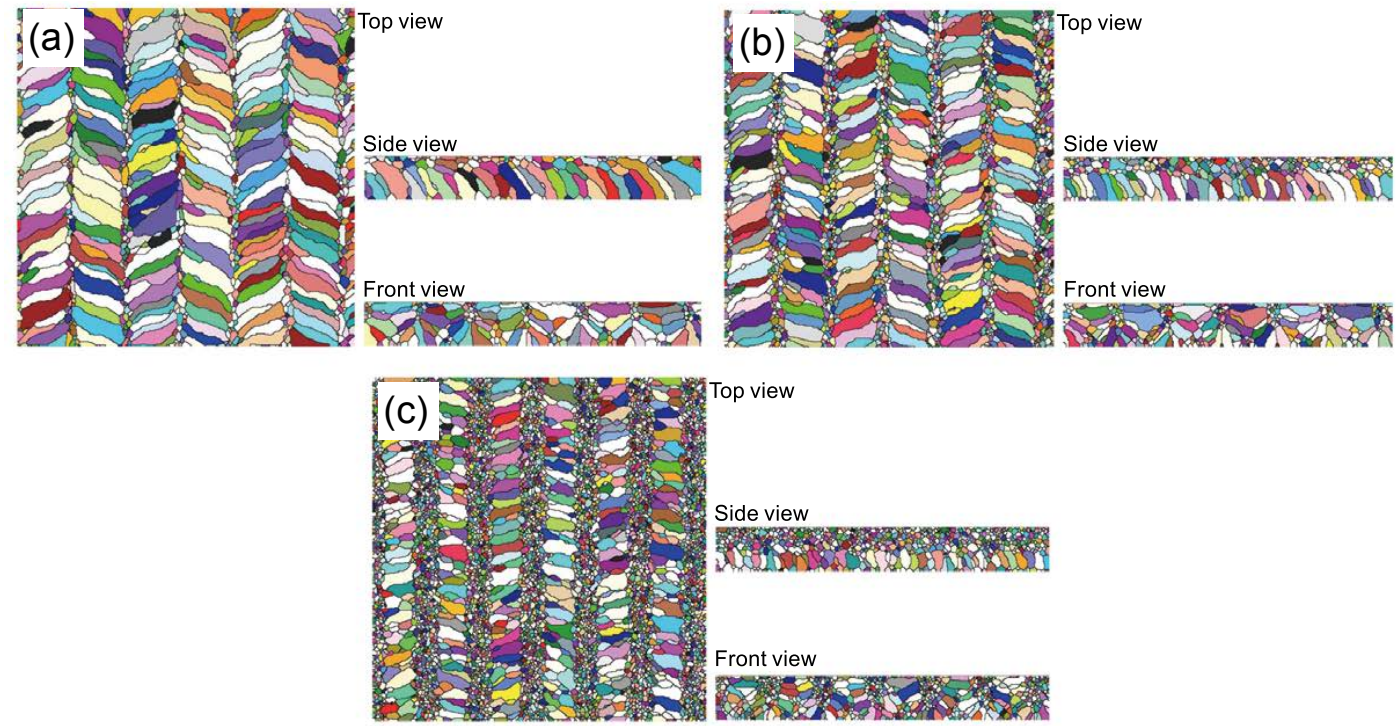

Fig. 18: Grain morphology at laser scanning speed of 450 (a), 600 (b), and 1,000 (c) $\mathrm{mm} \cdot \mathrm{min}^{-1}[6]$

model can be used to solve smaller problems, such as multilayer scanning.

Discrete powder particle heat flux coupling model focuses on using the physical behavior of single particle to simulate the complex molten pool evolution process. The movement of powder particles and spatter of molten pool are taken in consideration in this model. DEM makes the discretization degree of the model sharply increases. Therefore, the calculation load of the model is huge and only used to explore the formation mechanism of defects on powder scale.

Currently, the microstructure algorithm is mainly used to predict the solidification of primary phase and dendrite morphology.

The physical control equations in the phase field method are abundant, which can be used to predict the dendrite morphology with high accuracy. However, it is difficult to be used to solve the three-dimensional large-scale problems of metal additive manufacturing due to the large amount of calculation. At present, phase field method is mainly used to simulate the finite number of dendrites in two-dimensional model.

The CA method combines the stochastic algorithm based on the physical mechanism of nucleation with the theory of crystal growth. It has the advantages of small amount of calculation and high efficiency. It is mainly used to simulate the solidification of primary phase.

MC method is also a probability model based on the principle of minimum interface energy of grain boundary. Compared with CA method, MC method has higher calculation efficiency, but it still has some limitations. MC method cannot provide accurate information of crystal orientation. Besides, MC method needs to introduce external model to provide the information of temperature field and time step, which makes the calculation parameters complex.

\section{Outlook}

Heat flux coupling model solved by Euler equation is difficult to be used to simulate the thermal stress and deformation during additive manufacturing. Therefore, establishing the 'thermal-flow-solid' coupling model is an important research direction. 'Thermal-flow-solid' strong coupling model for powder size in metal additive manufacturing process based on grid free method and equivalent model coupling method (thermal conduct model-thermal flux flow model) are the development direction worthy of attention.

The macro manufacturing process and micro evolution process are quite different in time and space scale, the amount of calculation in the coupling process is very large, and the calculation error is easy to occur. Due to the large amount of calculation, the multi-scale coupling model is difficult to solve the problem of shape control and process parameter optimization in metal additive manufacturing.

There are many calculation models and large amount of calculation in numerical simulation of metal additive manufacturing. The combination of numerical computation and deep machine learning can fully excavate the data meaning in computation, and efficiently establish multi-scale and multi physical field coupling model to predict the microstructure and mechanical properties change during additive manufacturing process.

At present, the numerical simulation model of additive manufacturing can be realized in general commercial software, and some integral additive manufacturing calculation models are also introduced by commercial software (Ansys Additive). It can be expected that more calculation models will be introduced into general commercial software, which will become an effective tool for analyzing shape control process and optimizing process parameters of metal additive manufacturing.

\section{Acknowledgements}

This work was financially supported by the National Key R\&D Program of China (No. 2017YFE0123500 and No. 2017YFB1103701). 


\section{References}

[1] Maleki E, Bagherifard S, Bandini M, et al. Surface posttreatments for metal additive manufacturing: Progress, challenges, and opportunities. Additive Manufacturing, 2021, 37: 101619 .

[2] Hu Y B, Cong W L. A review on laser deposition-additive manufacturing of ceramics and ceramic reinforced metal matrix composites. Ceramics International, 2018, 44(17): 20599-20612.

[3] Esmail I, Sarvestani H Y, Gholipour J, et al. Engineered net shaping of alumina ceramics using picosecond laser. Optics and Laser Technology, 2020, 135, doi:10.1016/ j.optlastec.2020.106669.

[4] Ge J G, Ma T J, Han W T, et al. Thermal-induced microstructural evolution and defect distribution of wire-arc additive manufacturing $2 \mathrm{Cr} 13$ part: Numerical simulation and experimental characterization. Applied Thermal Engineering, 2019, 163, doi.org/10.1016/j.applthermaleng.2019.114335.

[5] Bi J, Lei Z L, Chen Y B, et al. Microstructure and mechanical properties of a novel Sc and $\mathrm{Zr}$ modified 7075 aluminum alloy prepared by selective laser melting. Materials Science and Engineering A, 2019, 768, doi: 10.1016/j.msea.2019.138478.

[6] Konyashin I, Hinners $\mathrm{H}$, Ries B, et al. Additive manufacturing of WC- $13 \%$ Co by selective electron beam melting: Achievements and challenges. International Journal of Refractory Metals \& Hard Materials, 2019, 84, doi.org/10.1016/ j.ijrmhm.2019.105028.

[7] $\mathrm{Li} \mathrm{Y} \mathrm{J,} \mathrm{Luo} \mathrm{Y,} \mathrm{Li} \mathrm{J} \mathrm{H,} \mathrm{et} \mathrm{al.} \mathrm{Ferrite} \mathrm{formation} \mathrm{and} \mathrm{its} \mathrm{effect} \mathrm{on}$ deformation mechanism of wire arc additive manufactured 308L stainless steel. Journal of Nuclear Materials, 2021, 550 , doi.org/10.1016/j.jnucmat.2021.152933.

[8] Razavykia A, Brusa E, Delprete C, et al. An overview of additive manufacturing technologies - A review to technical synthesis in numerical study of selective laser melting. Materials, 2020, 13(17): 3895.

[9] Tan J H K, Sing S L, Yeong W Y. Microstructure modelling for metallic additive manufacturing: A review. Virtual and Physical Prototyping, 2020, 15(1): 87-105.

[10] Ai Y W, Jiang P, Wang C M, et al. Experimental and numerical analysis of molten pool and keyhole profile during high-power deep-penetration laser welding. International Journal of Heat and Mass Transfer, 2018, 126: 779-789.

[11] Shi Q M, Gu D D, Xia M A, et al. Effects of laser processing parameters on thermal behavior and melting/solidification mechanism during selective laser melting of TiC/Inconel 718 composites. Optics \& Laser Technology, 2016, 84: 9-22.

[12] Dai D P, Jiang $X H$, Cai J P. Numerical simulation of temperature field and stress distribution in Inconel $718 \mathrm{Ni}$ base alloy induced by laser cladding. Chinese Journal of Lasers, 2015, 42(9): 1-8.

[13] Bian P Y, Shao X D, Du J L. Finite element analysis of thermal stress and thermal deformation in typical part during SLM. Applied Sciences, 2019, 9(11): 2231.

[14] Cao L, Yuan X F. Study on the numerical simulation of the SLM molten pool dynamic behavior of a nickel-based superalloy on the workpiece scale. Materials, 2019, 12(14): 2272.

[15] He X. Liquid metal expulsion during laser spot welding of 304 stainless steel. Journal of Physics, 2006, 39(3): 525-534.

[16] Wang R P. Numerical simulation of transient temperature field during laser keyhole welding of 304 stainless sheet. Optics and Laser Technology, 2011, 43(4): 870-873.

[17] Versteeg $\mathrm{H}$. An introduction to computational fluid dynamics. Englewood, Prentice Hall Press, 1996.

[18] Tryggvason G. Direct numerical simulations of gas-liquid multiphase flows. Cambridge, Cambridge University Press, 2011
[19] Gong S L. Weld pool dynamics in deep penetration laser welding Beijing China, Aviation Industry Press, 2018. (In Chinese)

[20] Jin C. Numerical simulation of welding process. Beijing China, Science Press, 2017. (In Chinese)

[21] Gao X D, Feng Y Z, Gui X Y, et al. Numerical simulation of effects of laser incident angle on transient behaviors of molten pool and keyhole during laser welding. Journal of Mechanical Engineering, 2020, 56(22): 83-89.

[22] Fang H Y, Meng Q G, Xu W L, et al. New general double ellipsoid heat source model. Science and Technology of Welding and Joining, 2005, 10(3): 361-368.

[23] Chen J W, Xiong F Y, Huang C Y, et al. Numerical simulation on metallic additive manufacturing. Scientia Sinica Physica, Mechanica and Astronomica, 2020, 50(9): 090007.

[24] Zeng P. Finite element analysis and applications. Beijing China, Tsinghua University Press, 2004.

[25] Abbaszadeh M, Honnige J R, Martina F, et al. Numerical investigation of the effect of rolling on the localized stress and strain induction for wire plus arc additive manufactured structures. Journal of Materials Engineering and Performance, 2019, 28(8): 4931-4942.

[26] Zhang Z, Farahmand P, Kovacevic R. Laser cladding of 420 stainless steel with molybdenum on mild steel A36 by a high power direct diode laser. Materials \& Design, 2016, 109: 686699.

[27] Cartalade A, Younsi A, Regnier E, et al. Simulations of phasefield models for crystal growth and phase separation. In: Proc. 2nd International Summer School on Nuclear Glass Wasteform: Structure, Properties and Long-Term Behavior, 2014, 7: 72-78.

[28] Feng J R, Dai K D, Zhou Q, et al. Formation of bonding interface in explosive welding - A molecular dynamics approach. Journal of Physics Condensed Matter, 2019, 31(41), doi: 10.1088/1361-648X/ab30d7.

[29] Li J H, Zhou X L, Brochu M, et al. Solidification microstructure simulation of Ti-6Al-4V in metal additive manufacturing: A review. Additive Manufacturing, 2020, 31: 100989.

[30] Zhang Z, Tan Z J, Yao X, et al. Numerical methods for microstructural evolutions in laser additive manufacturing. Computers \& Mathematics with Applications, 2019, 78(7): 2296-2307.

[31] Zhang S J, Guillemot G, Gandin C A, et al. A partitioned twostep solution algorithm for concurrent fluid flow and stressstrain numerical simulation in solidification processes. Computer Methods in Applied Mechanics and Engineering, 2019, 356: 294-324.

[32] Kundin J, Steinbach I. Comparative study of different anisotropy and potential formulations of phase-field models for dendritic solidification. Computational Materials Science, 2019, 170: 109197.

[33] Chen L, Wang C M, Xiong L D, et al. Microstructural, porosity and mechanical properties of lap joint laser welding for 5182 and 6061 dissimilar aluminum alloys under different place configurations. Materials \& Design, 2020, 191: 108625.

[34] Biner S. Programming phase-field modeling. Berlin, Springer Press, 2017

[35] Rappaz M, Gandin C A. Probabilistic modelling of microstructure formation in solidification processes. Acta Metallurgica Materialia, 1993, 41(2): 345-360.

[36] Jeong K, Kim B, Lee S J. Growth kinetics of the twodimensional Ising model with finite cooling rates. Physical Review E. 2019, 99(2), doi:10.1103/PhysRevE.99.022113.

[37] Babu B, Lundback A, Lindgren L E. Simulation of Ti-6Al-4V additive manufacturing using coupled physically based flow stress and metallurgical model. Materials, 2019, 12(23): 3844. 
[38] Bertini L, Bucchi F, Frendo F, et al. Residual stress prediction in selective laser melting - A critical review of simulation strategies. International Journal of Advanced Manufacturing Technology, 2019, 105(1-4): 609-636.

[39] Toyserkani E, Khajepour A, Corbin S. Three-dimensional finite element modeling of laser cladding by powder injection: Effects of powder feed rate and travel speed on the process. Journal of Laser Applications, 2003, 15(3): 153-160.

[40] Sih S, Barlow J W. The Prediction of the emissivity and thermal conductivity of powder beds. Particulate Science and Technology, 2004, 22(4): 427-440.

[41] Li Y L, Zhou K, Tan P, et al. Modeling temperature and residual stress fields in selective laser melting. International Journal of Mechanical Sciences. 2018, 136: 24-35.

[42] Li C, Denlinger E R, Gouge M F, et al. Numerical verification of an Octree mesh coarsening strategy for simulating additive manufacturing processes. Additive Manufacturing, 2019, 30(3): 100903.

[43] Wang X J. Formation mechanism of solidification cracking in laser welding on Al-Mg-Si alloy. Dissertation: Shanghai Jiao Tong University, 2015. (In Chinese)

[44] Liang R. Study on numerical simulation for laser welding by coupled thermo-hydro-mechanical method. Doctoral Dissertation, Shanghai Jiao Tong University, 2019. (In Chinese)

[45] Ogino Y, Asai S, Hirata Y. Numerical simulation of WAAM process by a GMAW weld pool model. Welding in the World, 2018, 62(2): 393-401.

[46] Zhang T L, Xu G, Shen Y T, et al. Numerical simulation of molten pool behavior of MIG-based additive manufacturing. Intelligent Computer and Applications, 2020, 10(5): 252-256.

[47] Yuan P P, Gu D D. Molten pool behaviour and its physical mechanism during selective laser melting of TiC/AISi10Mg nanocomposites: simulation and experiments. Journal of Physics D: Applied Physics, 2015, 48(3): 035303.

[48] Gusarov A V, Laoui T, Froyen L, et al. Contact thermal conductivity of a powder bed in selective laser sintering. International Journal of Heat and Mass Transfer, 2003, 46(6): 1103-1109.

[49] Shi Q M, Gu D D, Xia M J, et al. Effects of laser processing parameters on thermal behavior and melting/solidification mechanism during selective laser melting of TiC/Inconel 718 composites. Optics \& Laser Technology, 2016, 84: 9-22.

[50] Gan Z T, Liu H, Li S X, et al. Modeling of thermal behavior and mass transport in multi-layer laser additive manufacturing of Ni-based alloy on cast iron. International Journal of Heat and Mass Transfer, 2017, 111: 709-722.

[51] Khasenova R, Komarov S, Ishihara S, et al. Discrete element method simulations of mechanical plating of composite coatings on aluminum substrates. Surface \& Coatings Technology, 2018, 349: 949-958.
[52] Qu R Z, Huang L P, Xiao D M. Study on the numerical simulation of melt pool evolution and metal spattering characterization during selective laser melting processing. Acta Aeronautica et Astronautica Sinica, 2021, doi: 10.7527/S1000-6893.2021.25240.

[53] Chen Z, Xiang Y, Wei Z Y, et al. Thermal dynamic behavior during selective laser melting of K418 superalloy: numerical simulation and experimental verification. Applied Physics A, 2018, 124: 1-16.

[54] Krüger T. The lattice boltzmann method. Berlin, Springer Press, 2017.

[55] Zakirov A, Belousov S, Bogdanova M, et al. Predictive modeling of laser and electron beam powder bed fusion additive manufacturing of metals at the mesoscale. Additive Manufacturing, 2020, 35, doi.org/10.1016/j.addma.2020.101236.

[56] Fehn N, Heinz J, Wall W A, et al. High-order arbitrary LagrangianEulerian discontinuous Galerkin methods for the incompressible Navier-Stokes equations. Journal of Computational Physics, 2021, 430, doi.org/10.1016/j.jcp.2020.110040.

[57] John D A. Computational fluid dynamics -The basic with application. Beijing China, Tsinghua University Press, 2002.

[58] Khairallah S A, Anderson A T, Rubenchik A, et al. Laser powder-bed fusion additive manufacturing: Physics of complex melt flow and formation mechanisms of pores, spatter, and denudation zones. Acta Mater., 2016, 108: 36-45

[59] Farzadi A, Do-Quang M, Serajzadeh S, et al. Phase-field simulation of weld solidification microstructure in an $\mathrm{Al}-\mathrm{Cu}$ alloy. Modelling and Simulation in Materials Science and Engineering, 2008, 16: 1-18.

[60] Fallah V, Amoorezaei M, Provatas N, et al. Phase-field simulation of solidification morphology in laser powder deposition of $\mathrm{Ti}-\mathrm{Nb}$ alloys. Acta Materialia, 2012, 60(4): 1633-1646.

[61] Liu P W, Ji Y Z, Wang Z, et al. Investigation on evolution mechanisms of site-specific grain structures during metal additive manufacturing. Journal of Materials Processing Technology, 2018, 257: 191-202.

[62] Zhi Y. Application of cellular automata in the study of metal materials. Beijing China, Science Press, 2020.

[63] Kang $Y$, Zhan X H, Qi C Q, et al. Grain growth and texture evolution of weld seam during solidification in laser beam deep penetration welding of 2219 aluminum alloy. Materials Research Express, 2019, 6: 1-18.

[64] Mohebbi M S, Ploshikhin V. Implementation of nucleation in cellular automaton simulation of microstructural evolution during additive manufacturing of $\mathrm{Al}$ alloys. Additive Manufacturing, 2020, 36, doi.org/10.1016/j.addma.2020.101726.

[65] Baumgärtner A. Applications of the Monte Carlo method in statistical physics. Berlin, Springer Press, 1984.

[66] Li W, Soshi M. Modeling analysis of the effect of laser transverse speed on grain morphology during directed energy deposition process. The International Journal of Advanced Manufacturing Technology, 2019, 103(9-12): 3279-3291. 